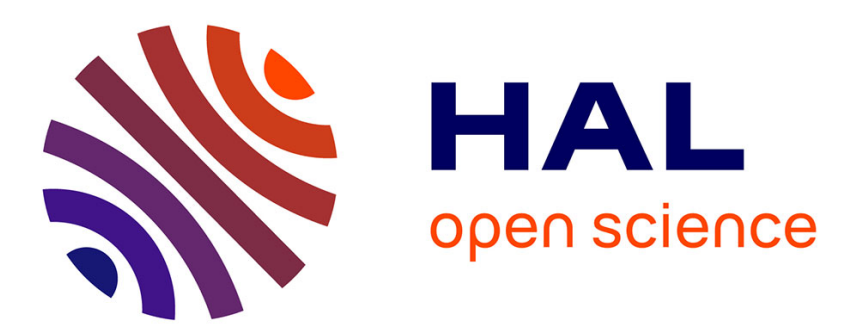

\title{
A Fictitious domain approach with spread interface for elliptic problems with general boundary conditions
}

\author{
Isabelle Ramière, Philippe Angot, Michel Belliard
}

\section{To cite this version:}

Isabelle Ramière, Philippe Angot, Michel Belliard. A Fictitious domain approach with spread interface for elliptic problems with general boundary conditions. Computer Methods in Applied Mechanics and Engineering, 2007, 196 (4-6), pp 766-781. hal-00274445

\section{HAL Id: hal-00274445 \\ https://hal.science/hal-00274445}

Submitted on 18 Apr 2008

HAL is a multi-disciplinary open access archive for the deposit and dissemination of scientific research documents, whether they are published or not. The documents may come from teaching and research institutions in France or abroad, or from public or private research centers.
L'archive ouverte pluridisciplinaire HAL, est destinée au dépôt et à la diffusion de documents scientifiques de niveau recherche, publiés ou non, émanant des établissements d'enseignement et de recherche français ou étrangers, des laboratoires publics ou privés. 


\title{
A fictitious domain approach with spread interface for elliptic problems with general boundary conditions
}

\author{
Isabelle Ramière ${ }^{\mathrm{a}, \mathrm{b}, *}$, Philippe Angot ${ }^{\mathrm{a}}$, Michel Belliard ${ }^{\mathrm{b}}$ \\ ${ }^{a}$ LATP-CMI, Technopôle de Château-Gombert, 13453 Marseille cedex 13, France \\ ${ }^{\mathrm{b}}$ CEA-Cadarache, DEN/DTN/SMTM/LMTR, 13108 St-Paul-Lez-Durance, France
}

\begin{abstract}
The aim of this article is to solve second-order elliptic problems in an original physical domain using a fictitious domain method with a spread interface approach. The main idea of the fictitious domain approach consists in immersing the original domain of study into a geometrically bigger and simpler one called fictitious domain. As the spatial discretization is being performed in the fictitious domain, this method allows the use of structured meshes. The discretization is not boundary-fitted to the original physical domain. This paper describes several ways to impose Dirichlet, Robin or Neumann boundary conditions on a spread immersed interface, without locally modifying the numerical scheme and without using Lagrange multipliers.

The numerical applications focus on diffusion and convection problems in the unit disk, with Dirichlet or Robin boundary conditions. For such problems, analytical solutions can be determined for a correctly chosen source term. The numerical resolution is performed using a $\mathcal{Q}_{1}$ Finite Element scheme. The spread interface approach is then combined with a local adaptive mesh refinement algorithm in order to increase the precision in the vinicity of the immersed boundary. The $L^{2}$ norm of the errors is computed in order to evaluate the capability of the method.

Immersed boundaries are found in many industrial applications like two-phase flow simulations, fluid/ structure interaction, etc. This article represents a first step towards the simulation of these kinds of applications.
\end{abstract}

Key words: Fictitious domain method, embedded boundary conditions, spread interface method, elliptic problems, local adaptive mesh refinement.

\footnotetext{
* Corresponding author.

Email address: ramiere@cmi.univ-mrs.fr (Isabelle Ramière).
} 


\section{Introduction}

Let $\tilde{\Omega}$ be an open bounded domain in $\mathbb{R}^{d}(d=2,3)$ with a boundary $\partial \tilde{\Omega}$ that is sufficiently regular. The resolution of a given problem $(\tilde{\mathcal{P}})$ in $\tilde{\Omega}$ with different kinds of boundary conditions (B.C.) is considered. When the shape of $\partial \tilde{\Omega}$ is geometrically complex, classical methods involving structured or unstructured boundary-fitted meshes induce a loss of efficiency and rapidity of numerical solvers in comparison with Cartesian meshes. Moreover, in the case of moving boundaries, the cost of mesh generation and re-meshing can be significant.

In the fictitious domain approach $[1,2]$, the original domain $\tilde{\Omega}$ is embedded in a geometrically bigger and simply-shaped other one $\Omega$, called fictitious domain (cf. Figure 1(a)).

The spatial discretization is now performed in $\Omega$, independently of the shape of the original domain $\tilde{\Omega}$. The original domain and the computational one are uncoupled. Numerical methods involving structured and Cartesian meshes can be used. The advantages of these methods are well known: natural tensor formulation, easy implementation for fast solvers (based for instance on finite volume methods with Cartesian grids) and multi-level methods [3], good convergence properties, etc. Consequently, the resolution of the new problem in $\Omega$ will be fast and simple.

The main issue lies in both the choice of the problem $(\mathcal{P})$ solved in the fictitious domain $\Omega$ and in the numerical scheme used for the resolution. These two choices have to be linked in order to handle the original boundary conditions on $\partial \tilde{\Omega}$. The B.C. on the original boundary $\partial \tilde{\Omega}$ must still be enforced so that the solution $u$ of the extended problem $(\mathcal{P})$ matches the solution $\tilde{u}$ of the original problem $(\tilde{\mathcal{P}})$ in the original domain $\tilde{\Omega}$.

The fictitious domain method was introduced in the sixties [1] and for a few years now, fictitious domain methods have been hugely developed and have arisen in different fields: computational fluid dynamics [4], medical simulation [5], etc. Numerically, there are two main approaches available to deal with the embedded boundary conditions on the immersed boundary:

- "Thin" interface approaches: the original boundary is approximated without being enlarged in the normal direction. The original boundary and the approximated one lie in the same $\mathbb{R}^{d-1}$ space. For example, the following methods can be found in this group: truncated domains methods [6,7], immersed interface methods (I.I.M.) [8,9], penalty methods $[1,2,10,11,12,4]$, fictitious domain methods with Lagrange multipliers [13,14,15], an adapted Galerkin method proposed in [16], and recent work on a fictitious model with flux and solutions jumps for general embedded boundary conditions $[17,18]$. 
- "Spread" interface approaches: the support of the approximated interface is larger than the original one. The approximated interface has one dimension more than the original one. For example, the spread interface can be a ring containing the immersed interface. This kind of approach can be found in $[2,19]$, in fluid/structure applications with the immersed boundary method (I.B.M.) [20,21], and more recently with the fat boundary method [22,23].

Numerous papers have been dedicated to embedded Dirichlet or Neumann B.C., e.g. $[1,2,8,10,15,6,24]$ and the references herein. Only a few studies have been devoted to embedded Fourier B.C. $[25,19,2,12,17,18]$.

The fictitious domain approach introduced here deals with Dirichlet, Robin and Neumann B.C. on an immersed interface without having to change the numerical scheme near the immersed interface or use Lagrange multipliers. Since the fictitious problem $(\mathcal{P})$ is not a saddle-point problem, the inf-sup condition does not need verifying (e.g. [15,24]). Moreover, only one discretization grid is used. The fictitious domain is meshed with a structured regular uniform grid.

The fictitious domain method presented in this article easily simulates freeboundary problems with possible boundary deformations without increasing the computational cost. A boundary non-conforming mesh is used, which conserves the first-order accuracy. The precision of the solution is improved by a local mesh refinement in the vicinity of the immersed interface.

A "spread" interface approach (see section 2.2) has been chosen here compared to our other works $[17,18]$ which deal with an approximated "thin" interface. In comparison with the original problem $(\tilde{\mathcal{P}})$, the terms of the fictitious problem $(\mathcal{P})$ solved all over the fictitious domain $\Omega$ are designed to handle the original B.C. of $(\tilde{\mathcal{P}})$. The restriction of the problem $(\mathcal{P})$ over the original domain $\tilde{\Omega}$ is chosen to be similar to $(\tilde{\mathcal{P}})$. Appropriate data in the external domain $\Omega_{e}$ and B.C. on $\partial \Omega$ have to be determined.

In the following sections, we will study the resolution of an original elliptic problem $(\tilde{\mathcal{P}})$, which can be either a diffusion or a convection-diffusion problem. The associated problem $(\mathcal{P})$ solved in the fictitious domain is introduced. Several ways to impose usual B.C. on a spread approximated immersed interface are then discussed. This fictitious domain method is then combined with a multi-grid local mesh refinement to increase the precision of the solution. The last section is dedicated to numerical resolution. A F.E. scheme is implemented and several results are provided to illustrate the accuracy of the method. 


\section{The fictitious domain method with spread interface for diffusion problems}

\subsection{Presentation of the original problem $(\tilde{\mathcal{P}})$}

For sake of simplicity we choose to focus on 2D problems, even though the formulations can be extended to cover 3D problems without any difficulty. The resolution of a diffusion-reaction problem $(\tilde{\mathcal{P}})$ in the original domain $\tilde{\Omega}$ is investigated.

Let us consider the following model problem: For $\tilde{\mathbf{a}} \in\left(L^{\infty}(\tilde{\Omega})\right)^{d \times d}, \tilde{b} \in\left(L^{\infty}(\tilde{\Omega})\right)$ and $\tilde{f} \in$ $L^{2}(\tilde{\Omega})$, find a function $\tilde{u}$ defined on $\tilde{\Omega}$ such that:

$$
(\tilde{\mathcal{P}}) \quad \begin{cases}-\operatorname{div}(\tilde{\mathbf{a}} \cdot \nabla \tilde{u})+\tilde{b} \tilde{u}=\tilde{f} & \text { in } \tilde{\Omega} \\ \text { B.C. } & \text { on } \partial \tilde{\Omega}\end{cases}
$$

where B.C. represents several types of boundary

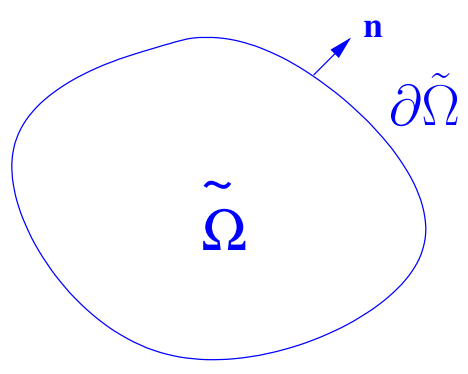
conditions:

- A Dirichlet condition: $\tilde{u}=u_{D}$ with $u_{D} \in H^{1 / 2}(\partial \tilde{\Omega})$,

- A Robin (or Fourier) condition: $-(\tilde{\mathbf{a}} \cdot \nabla \tilde{u}) \cdot \mathbf{n}=\alpha_{R} \tilde{u}+g_{R}$, with $\alpha_{R} \in L^{\infty}(\partial \tilde{\Omega}) ; \alpha_{R} \geqslant 0$, and $g_{R} \in L^{2}(\partial \tilde{\Omega})$ (with $\mathbf{n}$ the outward unit normal vector on $\partial \tilde{\Omega})$

Remark: a Neumann condition, $-(\tilde{\mathbf{a}} \cdot \nabla \tilde{u}) \cdot \mathbf{n}=g$, is considered as a particular Robin condition where $\alpha_{R} \equiv 0$ and $g_{R} \equiv g$.

Moreover, the tensor of diffusion $\tilde{\mathbf{a}} \equiv\left(\tilde{a}_{i j}\right)_{1 \leqslant i, j \leqslant d}$ and the reaction coefficient $\tilde{b}$ verify the classical ellipticity assumptions:

$$
\begin{gathered}
\exists \tilde{a}_{0}>0, \forall \boldsymbol{\xi} \in \mathbb{R}^{d}, \tilde{\mathbf{a}}(x) \cdot \boldsymbol{\xi} \cdot \boldsymbol{\xi} \geqslant \tilde{a}_{0}|\boldsymbol{\xi}|^{2} \text { a.e. in } \tilde{\Omega} \\
\text { where }|.| \text { is the Euclidean norm in } \mathbb{R}^{d} \\
\exists \tilde{b}_{0} \geqslant 0, \tilde{b}(x) \geqslant \tilde{b}_{0} \quad \text { a.e. in } \tilde{\Omega}
\end{gathered}
$$

In this case, classical variational techniques (e.g. [26,27]) prove that the solution $\tilde{u}$ of the original problem $(\tilde{\mathcal{P}})$ exists and is the unique solution $\tilde{u}$ in the space $H^{1}(\tilde{\Omega})$ satisfying the weak formulation of $(\tilde{\mathcal{P}})$. 


\subsection{Fictitious problem $(\mathcal{P})$}

The original domain $\tilde{\Omega}$ is embedded inside a fictitious domain $\Omega$ such that $\Omega=\tilde{\Omega} \cup \Sigma \cup \Omega_{e}$, where $\Omega_{e}$ is the external fictitious domain and $\Sigma$ the common interface between $\tilde{\Omega}$ and $\Omega_{e}$ (see Figure $1(\mathrm{a})$ and $4(\mathrm{~b})$ ). This original interface $\Sigma$ is called the immersed interface. The fictitious domain $\Omega$ is chosen to be geometrically simple (rectangular for example). If $\partial \tilde{\Omega} \cap \partial \Omega \neq \emptyset$ (see Figure4(b)), the boundary of $\tilde{\Omega}$ is defined by $\partial \tilde{\Omega}=\tilde{\Gamma} \cup \Sigma$, and the boundary of $\Omega$ by $\partial \Omega=\tilde{\Gamma} \cup \Gamma_{e}$. Otherwise (see Figure $\left.1(\mathrm{a})\right), \tilde{\Gamma} \equiv \emptyset$, so $\partial \tilde{\Omega} \equiv \Sigma$ and $\partial \Omega \equiv \Gamma_{e}$.

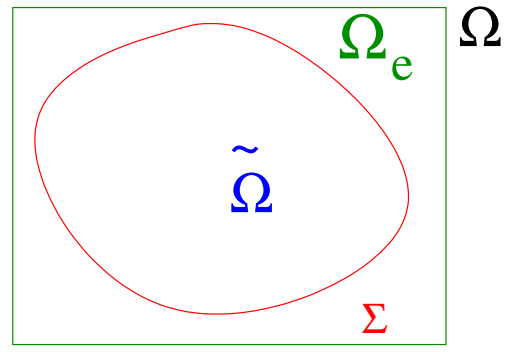

(a) Fictitious domain $\Omega$

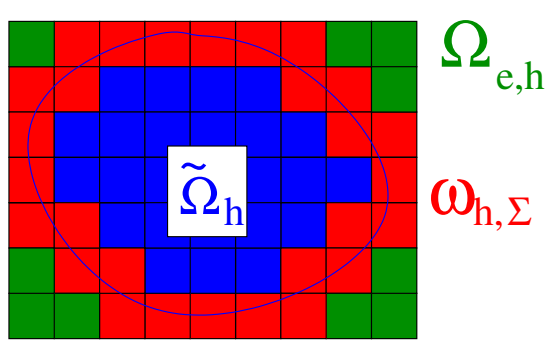

(b) Spread interface $\omega_{h, \Sigma}$

Figure 1. Example of an original domain $\tilde{\Omega}$ immersed in the fictitious rectangular domain $\Omega$. Discretization of the fictitious domain $\Omega: \Omega=\tilde{\Omega}_{h} \cup \omega_{h, \Sigma} \cup \Omega_{e, h}$

The computational domain $\Omega$ is uniformly meshed with a family $\mathcal{T}_{h}=\{K\}$ of disjointed rectangular cells $K$ such that $\bar{\Omega}=\underset{K \in \mathcal{T}_{h}}{\cup} \bar{K}$. A "spread" interface approach $[21,28]$ is chosen to approximate the immersed interface $\Sigma$. The approximated interface $\omega_{h, \Sigma}$ is the open bounded domain defined as $\omega_{h, \Sigma}=\bar{\omega}_{h, \Sigma} \backslash \partial \bar{\omega}_{h, \Sigma}$, where $\bar{\omega}_{h, \Sigma}=\underset{K \in \mathcal{T}_{h}}{\cup}\{\bar{K}, K \cap \Sigma \neq \emptyset\}$. (cf. Figure 1(b)). form:

The problem $(\mathcal{P})$ solved in the fictitious domain $\Omega$ has the following generic $0<\eta$ being a real parameter specified later, find $u_{\eta}^{h}$ (depending on the mesh $\mathcal{T}_{h}$ ) a real-valued function in $\Omega$ such that

$$
(\mathcal{P}) \quad \begin{cases}-\operatorname{div}\left(\mathbf{a} \cdot \nabla u_{\eta}^{h}\right)+b u_{\eta}^{h}=f & \text { in } \Omega \\ \text { original B.C. of }(\tilde{\mathcal{P}}) & \text { on } \tilde{\Gamma} \\ \text { suitable B.C. for } u_{\eta}^{h} & \text { on } \Gamma_{e}\end{cases}
$$

where $\mathbf{a} \in\left(L^{\infty}(\Omega)\right)^{d^{2}}, b \in\left(L^{\infty}(\Omega)\right), f \in L^{2}(\Omega)$ such that:

$$
\left.\mathbf{a}\right|_{\tilde{\Omega}_{h}}=\left.\tilde{\mathbf{a}}\right|_{\tilde{\Omega}_{h}},\left.\quad b\right|_{\tilde{\Omega}_{h}}=\left.\left.\tilde{b}\right|_{\tilde{\Omega}_{h}} \quad f\right|_{\tilde{\Omega}_{h}}=\left.\tilde{f}\right|_{\tilde{\Omega}_{h}},
$$

and $\mathbf{a}$ and $b$ respectively satisfy the assumptions (A1) and (A2) in $\Omega$. 
Once again, for B.C. correctly chosen on $\Gamma_{e}$ (such that the problem $(\mathcal{P})$ is well-posed), variational techniques [26,27] make it possible to conclude that the solution $u_{\eta}^{h}$ of the problem $(\mathcal{P})$ exists, is unique and belongs to $H^{1}(\Omega)$.

The extensions a, $b$ and $f$ of the original coefficients enable us to take account of the immersed boundary $[4,12,29]$. For each kind of boundary conditions lying on the immersed boundary $\Sigma$, different possibilities to enforce these conditions exist using a spread approximation of the immersed interface $\Sigma$ and are introduced in the next sections. No variant modifies the numerical scheme or introduces local unknowns. It is expected that: $\left.\left.u_{\eta}^{h}\right|_{\tilde{\Omega}_{h}} \simeq \tilde{u}\right|_{\tilde{\Omega}_{h}}$.

\subsection{Methodology and different variants}

For sake of clarity, it is assumed that $u_{D}, \alpha_{R}$ and $g_{R}$ are constant. The non-constant case can be treated just as easily. The non-constant data are then replaced by their extensions in $\omega_{h, \Sigma} \cup \Omega_{e, h}$ such that the traces on $\Sigma$ of the extensions are equal to the original data on $\Sigma$.

\subsubsection{Embedded Dirichlet B.C.}

The Dirichlet embedded B.C. are treated by volume penalization (see e.g. [30]).

\subsubsection{Spread interface penalization}

The first possibility to have a Dirichlet condition on $\Sigma$ is to penalize the Dirichlet value in the approximated interface $\omega_{h, \Sigma}$.

Let $0<\eta<<1$ be a real penalty parameter which is likely to tend to zero. The $\mathbf{L}^{2}$ penalty [30] consists in penalizing the reaction coefficient $b$ and the source term $f$ in the spread interface:

$$
b=\frac{1}{\eta}, \quad f=\frac{1}{\eta} u_{D} \quad \text { in } \omega_{h, \Sigma}
$$

Then, the penalty parameter enforces $u_{\eta}^{h} \simeq u_{D}$ in the approximated immersed boundary $\omega_{h, \Sigma}$. By defining the spread interface, it implies $u_{\eta}^{h} \simeq u_{D}$ on the original boundary $\Sigma$.

The $\mathbf{H}^{\mathbf{1}}$ penalty [30] is a particular case of $L^{2}$ penalty where the diffusion coefficient $\mathbf{a}$ is also penalized in $\omega_{h, \Sigma}\left(\mathbf{a}=\frac{1}{\eta} \mathbf{I} \mathbf{d}\right)$. In this case, the value of $u$ and the gradient $\nabla u$ are penalized.

With a penalization of the spread interface $\omega_{h, \Sigma}$, the equation solved in $\Omega_{e, h}$ has no influence on the solution obtained in the original domain. So in $\Omega_{e, h}$, 
the equation coefficients are arbitrary extensions of the original coefficients of $\tilde{\mathcal{P}}$ (as long as the whole problem $(\mathcal{P})$ in $\Omega$ is well-posed).

Concerning the B.C. on $\Gamma_{e}$, the Dirichlet B.C. $u_{\eta}^{h}=u_{D}$ must be imposed only on $\Gamma_{e} \cap \partial \omega_{h, \Sigma}$. The rest of the B.C. on $\Gamma_{e}$ can be arbitrary chosen.

Remark: If $\Gamma_{e} \cap \partial \omega_{h, \Sigma} \neq \emptyset$, the generalized Poincaré inequality [31] holds in $\omega_{h, \Sigma}$. The $H^{1}$ penalty required penalizing the diffusion coefficient a only. The reaction coefficient $b$ and the source term $f$ don't need to be penalized any further.

\subsubsection{Exterior penalization}

The second approach consists in penalizing $u_{\eta}^{h}$ at $u_{D}$ only in the external domain $\Omega_{e, h}$ (the approximated interface $\omega_{h, \Sigma}$ is not penalized). All the external nodes are penalized at $u_{D}$ so the solution tends to $u_{D}$ near the original immersed boundary $\Sigma$. In this case, the coefficients of the original problem $(\tilde{\mathcal{P}})$ are extended in $\tilde{\Omega}_{h} \cup \omega_{h, \Sigma}$.

As in the previous case, the $\mathbf{L}^{2}$ penalty is obtained by setting:

$$
b=\frac{1}{\eta}, \quad f=\frac{1}{\eta} u_{D} \quad \text { in } \Omega_{e, h}
$$

Seeing that in this case the B.C. $\left.u\right|_{\Gamma_{e}}=u_{D}$ holds on $\Gamma_{e}$, the Poincaré inequality in $\Omega_{e, h}$ makes it possible to obtain the $\mathbf{H}^{\mathbf{1}}$ penalty by penalizing $\left.\mathbf{a}\right|_{\Omega_{e, h}}=\frac{1}{\eta} \mathbf{I d}$ only. This approach is similar to the one introduced in $[1,2]$.

These two variants are summarized in Table 1.

\subsubsection{Embedded Robin B.C.}

\subsubsection{Theoretical aspect}

The Robin condition is treated in a different manner than the Dirichlet one. Let us consider an extended diffusion problem $\left(\mathcal{P}_{e}\right)$ of $(\tilde{\mathcal{P}})$ in $\Omega_{e}$ with a unique solution $u_{e} \in H^{1}\left(\Omega_{e}\right)$ such that $\left.u_{e}\right|_{\Sigma}=\left.\tilde{u}\right|_{\Sigma}$.

Let $\mathbf{a}$ and $b$ be $L^{\infty}$ - extension of $\tilde{\mathbf{a}}$ and $\tilde{b}$ respectively in $\Omega$ and $f$ a $L^{2}-$ extension of $\tilde{f}$ in $\Omega$ such that:

$$
\star \mathbf{a}=\left\{\begin{array}{ll}
\tilde{\mathbf{a}} & \text { in } \tilde{\Omega} \\
\mathbf{a}_{\mathbf{e}} & \text { in } \Omega_{e}
\end{array} \quad b=\left\{\begin{array}{ll}
\tilde{b} & \text { in } \tilde{\Omega} \\
b_{e} & \text { in } \Omega_{e}
\end{array} \quad \star f= \begin{cases}\tilde{f} & \text { in } \tilde{\Omega} \\
f_{e} & \text { in } \Omega_{e}\end{cases}\right.\right.
$$

We then define $u \in H^{1}(\Omega)$ such that :

$$
\left.u\right|_{\tilde{\Omega}}:=\tilde{u} \text { and }\left.u\right|_{\Omega_{e}}:=u_{e}
$$


By considering the transmission problem between $\tilde{\Omega}$ and $\Omega_{e}$, using the weak formulations of the subproblems defined in $\tilde{\Omega}$ and $\Omega_{e}$, the following can be expressed:

$\forall v \in H^{1}(\Omega)$

$$
\begin{array}{cc}
\int_{\tilde{\Omega}} \mathbf{a} \cdot \nabla u \cdot \nabla v \mathrm{dx}+\int_{\tilde{\Omega}} b v \mathrm{dx}-\int_{\Sigma}(\mathbf{a} \cdot \nabla u)^{-} \cdot \mathbf{n} v \mathrm{ds}-\int_{\tilde{\Gamma}}(\mathbf{a} \cdot \nabla u) \cdot \mathbf{n} v \mathrm{ds}=\int_{\tilde{\Omega}} f v \mathrm{dx} \quad \text { in } \tilde{\Omega} \\
\int_{\Omega_{e}} \mathbf{a} \cdot \nabla u \cdot \nabla v \mathrm{dx}+\int_{\Omega_{e}} b v \mathrm{dx}+\int_{\Sigma}(\mathbf{a} \cdot \nabla u)^{+} \cdot \mathbf{n} v \mathrm{ds}-\int_{\Gamma_{e}}(\mathbf{a} \cdot \nabla u) \cdot \mathbf{n} v \mathrm{ds}=\int_{\Omega_{e}} f v \mathrm{dx} & \text { in } \Omega_{e}
\end{array}
$$

where $\mathbf{n}$ denotes the outward normal unit vector either on $\tilde{\Gamma}$, on $\Gamma_{e}$, or on $\Sigma$. In the latter case, $\mathbf{n}$ is oriented from $\tilde{\Omega}$ to $\Omega_{e}$. Thus, $(\mathbf{a} . \nabla u)^{-} .\left.\mathbf{n}\right|_{\Sigma}$ and $(\mathbf{a} . \nabla u)^{+} .\left.\mathbf{n}\right|_{\Sigma}$ are the traces of $\left.(\mathbf{a} . \nabla u) \cdot \mathbf{n}\right|_{\tilde{\Omega}}$ and $\left.(\mathbf{a} . \nabla u) \cdot \mathbf{n}\right|_{\Omega_{e}}$ on each side of $\Sigma$ respectively. Therefore, the addition of the two formulations in $\tilde{\Omega}$ and $\Omega_{e}$ leads to:

$\int_{\Omega} \mathbf{a} \cdot \nabla u \cdot \nabla v \mathrm{dx}+\int_{\Omega} b u v \mathrm{dx}+\int_{\Sigma} \llbracket(\mathbf{a} \cdot \nabla u) \cdot \mathbf{n} \rrbracket_{\Sigma} v \mathrm{ds}-\int_{\partial \Omega}(\mathbf{a} \cdot \nabla u) \cdot \mathbf{n} v \mathrm{ds}=\int_{\Omega} f v \mathrm{dx}$

where $\llbracket(\mathbf{a} . \nabla u) \cdot \mathbf{n} \rrbracket_{\Sigma}=\left.(\mathbf{a} . \nabla u)^{+} \cdot \mathbf{n}\right|_{\Sigma}-\left.(\mathbf{a} . \nabla u)^{-} \cdot \mathbf{n}\right|_{\Sigma}$

Then:

$\int_{\Omega} \mathbf{a} \cdot \nabla u \cdot \nabla v \mathrm{dx}+\int_{\Omega} b u v \mathrm{dx}-\int_{\partial \Omega}(\mathbf{a} \cdot \nabla u) \cdot \mathbf{n} v \mathrm{ds}=\int_{\Omega} f v \mathrm{dx}-<\llbracket(\mathbf{a} \cdot \nabla u) \cdot \mathbf{n} \rrbracket_{\Sigma} \delta_{\Sigma}, v>$

where $\delta_{\Sigma}$ refers to the Dirac delta measure supported on $\Sigma$.

In the distribution sense, the following equation is obtained in $\Omega$ :

$$
-\operatorname{div}(\mathbf{a} \cdot \nabla u)+b u=f-\llbracket(\mathbf{a} \cdot \nabla u) \cdot \mathbf{n} \rrbracket_{\Sigma} \delta_{\Sigma}
$$

The jump of flux across $\Sigma$ can be interpreted as a measure source term carried by $\Sigma$.

In our case, the following expression is required

$$
-(\mathbf{a} . \nabla u)^{-} .\left.\mathbf{n}\right|_{\Sigma}=\left.\alpha_{R} u\right|_{\Sigma}+g_{R}
$$

It is chosen to impose $-(\mathbf{a} . \nabla u)^{+} .\left.\mathbf{n}\right|_{\Sigma}=0$ by setting $\left.\mathbf{a}\right|_{\Omega_{e}}=\eta \mathbf{I d}$, so that

$$
\llbracket(\mathbf{a} . \nabla u) \cdot \mathbf{n} \rrbracket_{\Sigma}=\left.\alpha_{R} u\right|_{\Sigma}+g_{R}
$$

In the end, the following is obtained:

$$
-\operatorname{div}\left(\mathbf{a} . \nabla u_{\eta}\right)+b u_{\eta}=f-\left[\alpha_{R} u_{\eta}+g_{R}\right] \delta_{\Sigma}
$$

However, with a Cartesian mesh on $\Omega$, the support of $\Sigma$ is not exactly defined. A characteristic parameter $\epsilon$ is introduced in order to approximate the measure $\delta_{\Sigma}$ supported by $\Sigma$ by mollifiers [32] $\delta_{\omega_{h, \Sigma}}$ on the spread interface $\omega_{h, \Sigma}$. The term $\delta_{\omega_{h, \Sigma}}$ is a discrete Dirac function on the spread interface. Here, this discrete delta function is roughly approximated by a crenel function in $\Omega\left(\delta_{\omega_{h, \Sigma}}=1\right.$ 
in $\omega_{h, \Sigma}$ and 0 elsewhere) whereas a smoothed approximation using interaction equations is performed in the I.B.M., e.g. [20,5,21].

The principle is the following:

$$
\int_{\Sigma}\left\{\alpha_{R} u_{\eta}+g_{R}\right\} \mathrm{ds}=\int_{\omega_{h, \Sigma}} \frac{\alpha_{R} u_{\eta}^{h}+g_{R}}{\epsilon} \mathrm{dx}
$$

The parameter $\epsilon$ can be estimated in several manners, some of which will be presented in the next paragraph.

The equation solved in $\Omega$ is:

$$
-\operatorname{div}\left(\mathbf{a} . \nabla u_{\eta}^{h}\right)+b u_{\eta}^{h}=f-\left[\frac{\alpha_{R}}{\epsilon} u_{\eta}^{h}+\frac{g_{R}}{\epsilon}\right] \delta_{\omega_{h, \Sigma}}
$$

The coefficients of the original problem $(\tilde{\mathcal{P}})$ are extended in $\tilde{\Omega}_{h} \cup \omega_{h, \Sigma}$. The coefficient of the problem $(\mathcal{P})$ are obtained by identifying the equation $(5)$ with the generic formulation of the problem $(\mathcal{P})$ (see Table 1 ).

As in the Dirichlet case with a spread interface penalization, only the B.C. of the nodes lying on $\Gamma_{e} \cap \partial \omega_{h, \Sigma}$ induce the solution in the original domain. These B.C. must be homogeneous Neumann B.C. in order to have an external flux equal to zero.

Remark: Numerical schemes allowing local jumps of the solution u make it possible to also simulate a Robin immersed B.C. (e.g. [12,18,17]).

\subsubsection{Numerical aspect}

There are many ways to estimate the parameter $\epsilon$. This paper presents three of them representing different kinds of approximations (see $[28,33])$. If $h$ is the discretization step, Angot [29] showed that $\epsilon$ is in $\mathcal{O}(h)$.

- A coarse global approximation of $\epsilon$ in Equation (4) holds:

$$
\int_{\Sigma} \mathrm{ds}=\int_{\omega_{h, \Sigma}} \frac{1}{\epsilon} \mathrm{dx}
$$

* In a first approach, it is assumed that $\epsilon$ is constant all over $\omega_{h, \Sigma}$.

$$
\epsilon=\frac{\operatorname{meas}\left(\omega_{h, \Sigma}\right)}{\operatorname{meas}(\Sigma)}
$$

* In the second approach, the value of $\epsilon$ is given element by element. In the equation (4), the integration in $\omega_{h, \Sigma}$ is weighted by a coefficient $\tau$. This coefficient represents the presence rate of the original domain in each element $K$ crossed by the boundary $\Sigma\left(K \subset \omega_{h, \Sigma}\right)$. By construction, $\tau$ is constant on each $K$ :

$$
\tau_{K}=\frac{\text { volume of } \tilde{\Omega} \text { included in } K}{\text { volume of the element } K}
$$


By this way, the right hand side of (6) is only integrated in the original domain included in $\omega_{h, \Sigma}$. Introducing $\epsilon=\frac{\epsilon^{\prime}}{\tau}$ with a constant $\epsilon^{\prime}$ the following is obtained:

$$
\begin{aligned}
\int_{\Sigma} d s & =\int_{\omega_{h, \Sigma}} \frac{1}{\epsilon^{\prime}} \tau d x \\
\int_{\Sigma} d s & =\sum_{\left\{K \subset \omega_{h, \Sigma}\right\}} \int_{K} \frac{1}{\epsilon^{\prime}} \tau_{K} d x
\end{aligned}
$$

$\tau_{K}$ being constant on $K$ :

$$
\begin{aligned}
\epsilon^{\prime} & =\frac{\sum_{K}\left[\tau_{K} \cdot \operatorname{meas}(K)\right]}{\operatorname{meas}(\Sigma)} \\
\epsilon_{K} & =\frac{\sum_{K}\left[\tau_{K} \cdot \operatorname{meas}(K)\right]}{\tau_{K} \cdot \operatorname{meas}(\Sigma)}
\end{aligned}
$$

- A local approximation of $\epsilon$ consists in calculating $\epsilon$ in each cell $K \subset \omega_{h, \Sigma}$ :

$$
\int_{\Sigma_{K}} \mathrm{ds}=\int_{K} \frac{1}{\epsilon} \mathrm{dx}
$$

with $\Sigma_{K}=\Sigma \cap K$

As $\Sigma_{K}$ is not simply defined, the boundary $\Sigma$ is piecewise linear approximated by a segment $\Sigma_{l, K}$ in each cell $K \subset \omega_{h, \Sigma}$ (see Figure 2).

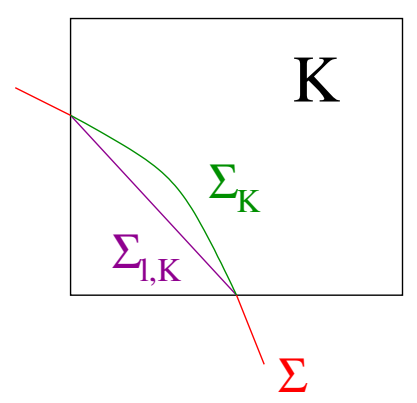

Figure 2. Linear approximation of $\Sigma$ in a rectangular cell $K \subset \omega_{h, \Sigma}$

The equation (9) is written using the linear approximation $\Sigma_{l}=\underset{K \in \omega_{h, \Sigma}}{\cup} \Sigma_{l, K}$ of $\Sigma$ :

Finally,

$$
\int_{\Sigma_{l, K}} \mathrm{ds} \simeq \int_{K} \frac{1}{\epsilon} \mathrm{dx}
$$

$$
\epsilon_{K}=\frac{\operatorname{meas}(K)}{\operatorname{meas}\left(\Sigma_{l, K}\right)}
$$

Here again, the value of $\epsilon$ depends on the element $K \subset \omega_{h, \Sigma}$ under consideration.

This approach induces a local piecewise linear reconstruction of the interface in each cell $K$ of the mesh. 
For each embedded B.C. variant, Table 1 gives the parameters of interest using a spread interface approach.

\section{Parameters in $\omega_{h, \Sigma} \quad$ Parameters in $\Omega_{e, h}$}

Dirichlet B.C.

$\begin{array}{rl}\text { Spread interface penalization } \quad \mathbf{a}=\left\{\begin{array}{l}\tilde{\mathbf{a}}\left(L^{2} \text { penalty }\right) \\ \frac{1}{\eta} \mathbf{I d}\left(H^{1} \text { penalty }\right)\end{array}, \quad \mathbf{a}=\mathbf{I d},\right. & \\ b=\frac{1}{\eta}, f=\frac{1}{\eta} u_{D} & b=0, f=0\end{array}$

Dirichlet B.C.

\begin{tabular}{c}
$\mathbf{a}=\tilde{\mathbf{a}}, \quad \mathbf{a}=\left\{\begin{array}{l}\tilde{\mathbf{a}}\left(L^{2} \text { penalty }\right) \\
\frac{1}{\eta} \mathbf{I d}\left(H^{1} \text { penalty }\right) \\
\text { Exterior penalization }, \\
b=\tilde{b}, f=\tilde{f}\end{array} \quad b=\frac{1}{\eta}, f=\frac{1}{\eta} u_{D}\right.$ \\
\hline \hline
\end{tabular}

Robin B.C.

with different approximations of $\epsilon$

$$
\begin{array}{cc}
\mathbf{a}=\tilde{\mathbf{a}}, & \mathbf{a}=\eta \mathbf{I} \mathbf{d}, \\
b=\tilde{b}+\frac{\alpha_{R}}{\epsilon}, f=\tilde{f}-\frac{g_{R}}{\epsilon} & b=0, f=0
\end{array}
$$

Table 1

Parameters in $\omega_{h, \Sigma}$ and in $\Omega_{e, h}$ for diffusion problems

\section{The fictitious domain method with spread interface for convection- diffusion problems}

\subsection{Presentation of the study}

Let us consider the following convection-diffusion-reaction problem: For $\tilde{\mathbf{a}} \in\left(L^{\infty}(\tilde{\Omega})\right)^{d \times d}, \quad \tilde{\mathbf{v}} \in\left(L^{\infty}(\tilde{\Omega})\right)^{d}, \quad \tilde{b} \in\left(L^{\infty}(\tilde{\Omega})\right)$ and $\tilde{f} \in L^{2}(\tilde{\Omega})$, find $\tilde{u} \in H^{1}(\tilde{\Omega})$ such that :

$$
(\tilde{\mathcal{P}}) \quad \begin{cases}-\operatorname{div}(\tilde{\mathbf{a}} \cdot \nabla \tilde{u})+\operatorname{div}(\tilde{\mathbf{v}} \tilde{u})+\tilde{b} \tilde{u}=\tilde{f} & \text { in } \tilde{\Omega} \\ \text { B.C. } & \text { on } \partial \tilde{\Omega}\end{cases}
$$

where B.C. represents Dirichlet, Robin or Neumann boundary conditions (see section 2). The diffusion tensor $\tilde{\mathbf{a}} \equiv\left(\tilde{a}_{i j}\right)_{1 \leqslant i, j \leqslant d}$ and the reaction coefficient $\tilde{b}$ verify the classical ellipticity assumptions $(A 1)$ and $(A 2)$. 
This problem needs to be solved using the fictitious domain method with spread interface introduced in the last section for diffusion problems. With an original convection-diffusion problem $(\tilde{\mathcal{P}})$ in $\tilde{\Omega}$, the problem $(\mathcal{P})$ solved in the fictitious domain $\Omega$ has the following generic form:

With $0<\eta \ll 1$, find $u_{\eta}^{h} \in H^{1}(\Omega)$ such that

$$
(\mathcal{P}) \quad \begin{cases}-\operatorname{div}\left(\mathbf{a} \cdot \nabla u_{\eta}^{h}\right)+\operatorname{div}\left(\mathbf{v} u_{\eta}^{h}\right)+b u_{\eta}^{h}=f & \text { in } \Omega \\ \text { original B.C. of }(\tilde{\mathcal{P}}) & \text { on } \tilde{\Gamma} \\ \text { suitable B.C. for } u_{\eta}^{h} & \text { on } \Gamma_{e}\end{cases}
$$

where $\mathbf{a} \in\left(L^{\infty}(\Omega)\right)^{d^{2}}, \mathbf{v} \in\left(L^{\infty}(\Omega)\right)^{d}, b \in\left(L^{\infty}(\Omega)\right)$, and $f \in L^{2}(\Omega)$ such that:

$$
\left.\mathbf{a}\right|_{\tilde{\Omega}_{h}}=\left.\tilde{\mathbf{a}}\right|_{\tilde{\Omega}_{h}},\left.\quad \mathbf{v}\right|_{\tilde{\Omega}_{h}}=\left.\tilde{\mathbf{v}}\right|_{\tilde{\Omega}_{h}},\left.\quad b\right|_{\tilde{\Omega}_{h}}=\left.\left.\tilde{b}\right|_{\tilde{\Omega}_{h}} \quad f\right|_{\tilde{\Omega}_{h}}=\left.\tilde{f}\right|_{\tilde{\Omega}_{h}},
$$

and $\mathbf{a}$ and $b$ satisfy classical ellipticity assumptions in $\Omega$.

With the spread interface approach, the embedded B.C. will be imposed using a unique Cartesian mesh and without locally modifying the numerical scheme.

\subsection{Treatment of the original B.C.}

\subsubsection{Embedded Dirichlet B.C.}

The Dirichlet B.C. are treated by volume penalization as is the case for diffusion problems (see section 2.3.1). We suggest comparing the penalization of the spread interface with the penalization of the exterior domain.

\subsubsection{Embedded Robin or Neumann B.C.}

As developed in section 2.3.2, the transmission problem between $\tilde{\Omega}$ and $\Omega_{e}$ with continuity of the solution on $\Sigma$ is taken into consideration. In the distribution sense, the following equation is obtained:

$$
-\operatorname{div}(\mathbf{a} \cdot \nabla u)+\operatorname{div}(\mathbf{v} u)+b u=f-\llbracket(\mathbf{a} \cdot \nabla u) \cdot \mathbf{n} \rrbracket_{\Sigma} \delta_{\Sigma}+\llbracket(\mathbf{v} \cdot \mathbf{n}) \rrbracket_{\Sigma} u \delta_{\Sigma}
$$

where $\left\{\begin{array}{l}\mathbf{n} \text { denotes the outward normal unit vector on } \Sigma\left(\text { oriented from } \tilde{\Omega} \text { to } \Omega_{e}\right) \\ \llbracket(\mathbf{a} \cdot \nabla u) \cdot \mathbf{n} \rrbracket_{\Sigma}=\left.(\mathbf{a} \cdot \nabla u) \cdot \mathbf{n}\right|_{\Sigma} ^{+}-\left.(\mathbf{a} \cdot \nabla u) \cdot \mathbf{n}\right|_{\Sigma} ^{-}=\left.\left(\mathbf{a}_{e} \cdot \nabla u\right) \cdot \mathbf{n}\right|_{\Sigma}-\left.(\tilde{\mathbf{a}} \cdot \nabla u) \cdot \mathbf{n}\right|_{\Sigma} \\ \llbracket(\mathbf{v} \cdot \mathbf{n}) \rrbracket_{\Sigma}=\left.\mathbf{v}_{e} \cdot \mathbf{n}\right|_{\Sigma}-\left.\tilde{\mathbf{v}} \cdot \mathbf{n}\right|_{\Sigma} \\ \delta_{\Sigma} \text { means the Dirac delta measure supported by } \Sigma\end{array}\right.$

Here again, the jump of fluxes (diffusion and convection) across $\Sigma$ appear as source terms carried by $\Sigma$. 
The following Robin flux is required

$$
-\left.(\mathbf{a} \cdot \nabla u) \cdot \mathbf{n}\right|_{\Sigma} ^{-}=-\left.(\tilde{\mathbf{a}} \cdot \nabla u) \cdot \mathbf{n}\right|_{\Sigma}=\left.\alpha_{R} u\right|_{\Sigma}+g_{R}
$$

We impose $-\left.(\mathbf{a} \cdot \nabla u) \cdot \mathbf{n}\right|_{\Sigma} ^{+}=0$ by $\mathbf{a}_{e}=\eta \mathbf{I d}$ on $\Omega_{e}$, so that

$$
\llbracket(\mathbf{a} \cdot \nabla u) \cdot \mathbf{n} \rrbracket_{\Sigma}=\left.\alpha_{R} u\right|_{\Sigma}+g_{R}
$$

Moreover, $\left.\mathbf{v}\right|_{\Omega_{e}}=\mathbf{v}_{e}=\mathbf{0}$ is imposed in order to free up the solution in the exterior domain $\Omega_{e}$. If $\mathbf{v}_{e} \neq \mathbf{0}$, as $\left.u\right|_{\Sigma} ^{-}=\left.u\right|_{\Sigma} ^{+}$, the resolution of the exterior problem influences the embedded Robin condition.

On $\Omega$, then we have:

$$
-\operatorname{div}\left(\mathbf{a} . \nabla u_{\eta}\right)+\operatorname{div}\left(\mathbf{v} u_{\eta}\right)+b u_{\eta}=f-\left[\alpha_{R} u_{\eta}+g_{R}+(\mathbf{v} \cdot \mathbf{n})^{-} u_{\eta}\right] \delta_{\Sigma}
$$

A characteristic parameter (see section 2.3 .2 and [32]) is introduced in order to approximate the Dirac function on $\Sigma$ by a discrete Dirac delta function in $\omega_{h, \Sigma}$. The principle is the following:

$$
\int_{\Sigma}\left\{\alpha_{R} u_{\eta}+g_{R}+(\tilde{\mathbf{v}} \cdot \mathbf{n})^{-} u_{\eta}\right\} \mathrm{ds}=\int_{\omega_{h, \Sigma}} \frac{\alpha_{R} u_{\eta}^{h}+g_{R}+(\mathbf{v} \cdot \mathbf{n}) u_{\eta}^{h}}{\epsilon} \mathrm{dx}
$$

where $\mathbf{v}$ is an extension of the velocity field $\tilde{\mathbf{v}}$ in $\tilde{\Omega}_{h} \cup \omega_{h, \Sigma}$ and $\mathbf{n}$ denotes either the outward unit vector on $\Sigma$ or its extension in $\omega_{h, \Sigma}$.

Next, the coefficients of the fictitious problem $(\mathcal{P})$ are easily set by extending the coefficients of the original problem $(\tilde{\mathcal{P}})$ in $\tilde{\Omega}_{h} \cup \omega_{h, \Sigma}$ and using the formulations (12) and (13) (see Table 2).

Only the B.C. on $\Gamma_{e} \cap \partial \omega_{h, \Sigma}$ has an effect on the solution obtained in the original domain. These B.C. must be homogeneous Neumann B.C. to vanish the external diffusion flux. The B.C. on the rest of $\Gamma_{e}$ can be arbitrarily chosen as long as the whole problem $(\mathcal{P})$ in $\Omega$ is well-posed.

Approximations of the parameter $\epsilon$ with Cartesian uniform meshes are obtained in the same manner as that in section 2.3.2.

For each embedded B.C. variant, Table 2 summarizes the parameters of interest for the resolution of a convection-diffusion problem.

\section{Spread interface approach and local adaptive mesh refinement}

Using a spread interface approach, Adaptive Mesh Refinement (AMR) techniques are necessary to improve the accuracy of the solution near the 


\section{Parameters in $\omega_{h, \Sigma} \quad$ Parameters in $\Omega_{e, h}$}

Dirichlet B.C.

Spread interface

penalization

$$
\begin{aligned}
& \mathbf{a}=\left\{\begin{array}{l}
\tilde{\mathbf{a}}\left(L^{2} \text { penalty }\right) \\
\frac{1}{\eta} \mathbf{I d}\left(H^{1} \text { penalty }\right)
\end{array}, \mathbf{v}=\tilde{\mathbf{v}}, \quad \mathbf{a}=\mathbf{I d}, \mathbf{v}=\mathbf{0},\right. \\
& b=\frac{1}{\eta}, f=\frac{1}{\eta} u_{D} \quad b=0, f=0
\end{aligned}
$$

Dirichlet B.C.

\begin{tabular}{cc} 
Exterior penalization $\quad \mathbf{a}=\tilde{\mathbf{a}}, \mathbf{v}=\tilde{\mathbf{v}}$, & $\mathbf{a}=\left\{\begin{array}{c}\tilde{\mathbf{a}}\left(L^{2} \text { penalty }\right) \\
\frac{1}{\eta} \mathbf{I d}\left(H^{1} \text { penalty }\right) \\
b=\tilde{b}, f=\tilde{f}\end{array}, \mathbf{v}=\mathbf{0}\right.$, \\
\hline \hline
\end{tabular}

Robin B.C.

$$
\begin{array}{cc}
\mathbf{a}=\tilde{\mathbf{a}}, \mathbf{v}=\tilde{\mathbf{v}}, & \mathbf{a}=\eta \mathbf{I} \mathbf{d}, \mathbf{v}=\mathbf{0}, \\
b=\tilde{b}+\frac{\alpha_{R}}{\epsilon}+\frac{\mathbf{v} \cdot \mathbf{n}}{\epsilon}, f=\tilde{f}-\frac{g_{R}}{\epsilon} & b=0, f=0
\end{array}
$$

Table 2

Parameters in $\omega_{h, \Sigma}$ and in $\Omega_{e, h}$ for convection-diffusion problems

immersed interface and by the way on the whole original domain. Most of these techniques are derived from multi-grid methods [34].

\subsection{Multi-grid method}

In order to simply present the multi-grid method, two nested grids only are taken into consideration: a "coarse" grid $G_{l}$ with a discretization step $h_{l}$ and a "fine" grid $G_{l+1}$ such that $h_{l+1}<h_{l}$. Generally speaking, a two-grid scheme can be divided into two steps:

- A descent or prolongation step from a coarse grid $G_{l}$ to a fine grid $G_{l+1}$. At this stage, an estimation of the solution on the coarse grid has already been calculated. This step consists in establishing boundary conditions on the boundary $\Gamma_{l+1}$ of the fine grid by interpolation and in solving the discrete problem associated with the grid $G_{l+1}$.

- An ascent or restriction step from a fine grid $G_{l+1}$ to a coarse grid $G_{l}$. The solution on the coarse grid $G_{l}$ is corrected according to the solution obtained on $G_{l+1}$ at the descent step. This correction can be either a simple local restriction on the solution or a local restriction on the defect. In the second case, an error equation is then solved on all the coarse grid $G_{l}$. 
The multi-grid method is a generalization of the two-grid scheme. If the solution obtained on a grid $G_{l+1}$ isn't precise enough, a two-grid scheme is performed again from $G_{l+1}$. A local fine grid $G_{l+2}$ such that $h_{l+2}<h_{l+1}$ is introduced. This procedure is repeated until the finest grid is reached. A recursive process is then generated.

For example, an iteration can be represented by a V-cycle: see Figure 3.

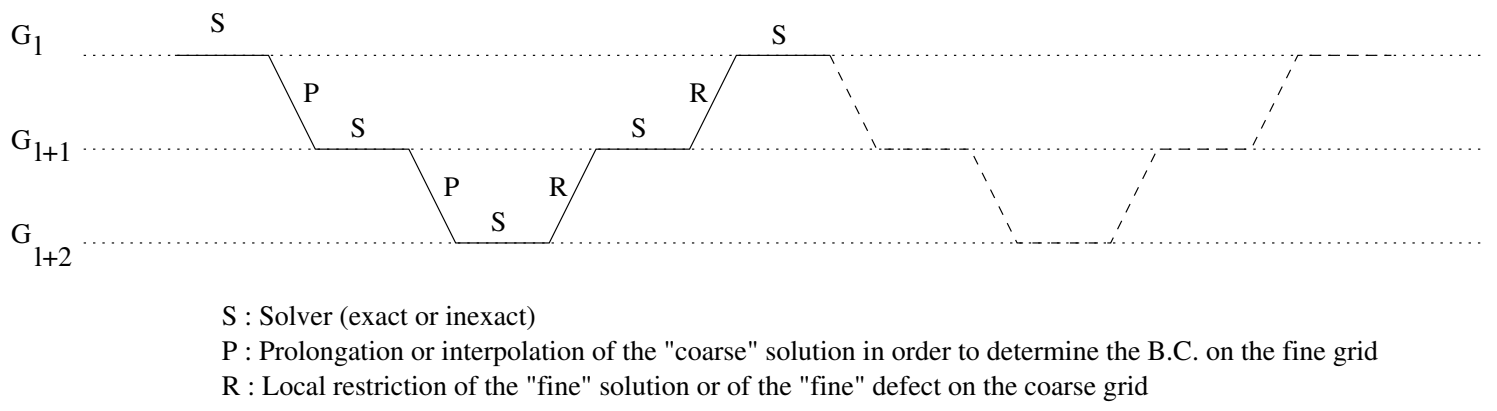

Figure 3. Representation of two V-cycles on three grids

The solver used on each grid is independent of the multi-grid method which has been chosen.

\subsection{Local Defect Correction (L.D.C.) method}

This method was introduced by Hackbusch [35]. This method is a multi-grid method with a defect restriction in the restriction step. The defect restriction is computed only on the coarse "interior" nodes. These nodes can be defined as the coarse nodes strictly included in the fine grid. In other words, this covers all the coarse nodes included in the fine grid except those lying on the fine grid boundary. The set of the coarse inner nodes associated with the grid $G_{l}$ is called $\mathcal{A}_{l}$.

Let us consider the resolution of the following problem:

$$
(\mathcal{P}) \begin{cases}L u=f(x) & \text { in } \Omega \\ \left.u\right|_{\Gamma} & \text { on } \Gamma=\partial \Omega\end{cases}
$$

At each iteration $\mathrm{k}$ of a multi-grid algorithm, the following discrete problem on the grid $G_{l}$ is solved:

$$
\left(\mathcal{P}_{l}^{k}\right)\left\{\begin{array}{l}
L_{l}^{k} u_{l}^{k}=f_{l}^{k} \quad \text { in } \Omega_{l} \\
\text { appropriate B.C. on } \Gamma_{l}
\end{array}\right.
$$


It is assumed that:

$\star L_{l}^{k}=\left.L\right|_{\Omega_{l}}$,

$\star f_{l}^{k=0}=\left.f\right|_{\Omega_{l}}$,

$\star \Omega_{0}=\Omega$.

The B.C. on $\Gamma_{l}$ can be divided into two parts:

$\star$ If $\Gamma_{l} \cap \Gamma \neq 0$, the B.C. on $\Gamma_{l} \cap \Gamma$ are computed by restricting on $\Gamma_{l}$ the B.C. lying on $\Gamma$,

* Otherwise the B.C. on $\Gamma_{l}$ are Dirichlet conditions obtained by interpolating the solution $u_{l-1}^{k}$.

For two grids $G_{l}$ and $G_{l+1}$, the L.D.C. algorithm writes:

Initialization: Computation of $u_{l}^{0}$, solution of the initial problem $\left(\mathcal{P}_{l}^{0}\right)$ on the grid $G_{l}$.

Iterations: Computation of $u_{l}^{k}$.

for $\mathrm{k}=1$ to nbcycles do $u_{l}^{k}=u_{l}^{k-1}$.

Resolution on the fine grid $G_{l+1}$ :

- Compute B.C. on $\Gamma_{l+1} \backslash\left(\Gamma_{l+1} \cap \Gamma\right)$ by interpolation of $u_{l}^{k}$ :

$$
u_{l+1}^{k}=P_{l+1}^{l} u_{l}^{k} \text { on } \Gamma_{l+1} \backslash\left(\Gamma_{l+1} \cap \Gamma\right)
$$

where $P_{l+1}^{l}$ is a interpolation operator also called coarse-to-fine operator.

- Computation of $u_{l+1}^{k}$ by solving $\left(\mathcal{P}_{l+1}^{k}\right)$.

Correction on the coarse grid $G_{l}$ :

- Restriction of the "fine" solution on $A_{l}$ :

$$
\tilde{u}_{l}^{k}(x)=\left(R_{l}^{l+1} u_{l+1}^{k}\right)(x) \forall x \in A_{l}
$$

where $R_{l}^{l+1}$ is a restriction operator also called fine-to-coarse operator.

- Computation of the local defect $r_{l}^{k}(\tilde{u})(x)=\left(f_{l}^{0}-L_{l} \tilde{u}_{l}^{k}\right)(x) \forall x \in A_{l}$.

- Computation of the corrected solution $u_{l}^{k}$ : resolution of the initial problem $\left(\mathcal{P}_{l}^{k}\right)$ with $f_{l}^{k}=f_{l}^{0}-\chi_{A_{l}} r_{l}^{k}(\tilde{u})$ (where $\chi$ is the characteristic function).

endfor

Remark: The "initial" L.D.C algorithm [34,3] introduces a subset $\mathcal{A}^{0}$ of $\mathcal{A}$ in order to evaluate the restricted defect according to nodes of $\mathcal{A}$ only. In this case, the refinement zones must be large enough to contain sufficient inner nodes. In our problem, this improvement is not necessary. The local defect restriction on $\mathcal{A}$ is accurate enough. 


\section{$5 \quad$ Numerical examples}

All the simulations have been computed thanks to the finite element industrial code PYGENE [36,37] of the Neptune project [38]. The Neptune project, co-developed by CEA and EDF, is dedicated to the simulation of two-phase flows in Nuclear Power Plants.

\subsection{Test problems}

The resolution of a diffusion problem and a convection-diffusion problem in the unit disk $\tilde{\Omega}$ is investigated. For symmetry reasons, this problem can only be solved on a quarter of this disk (see Figure 4(a)). The generic formulation of the problem $(\tilde{\mathcal{P}})$ under study writes:

$$
(\tilde{\mathcal{P}}) \quad \begin{cases}-\operatorname{div}(\tilde{\mathbf{a}} \cdot \nabla \tilde{u})+\operatorname{div}(\tilde{\mathbf{v}} \tilde{u})=\tilde{f} & \text { in } \tilde{\Omega} \\ (\tilde{\mathbf{a}} \cdot \nabla \tilde{u}) \cdot \mathbf{n}=0 & \text { on } \tilde{\Gamma} \\ \tilde{u}=u_{D} \text { or }-(\tilde{\mathbf{a}} \cdot \nabla \tilde{u}) \cdot \mathbf{n}=\alpha_{R} \tilde{u}+g_{R} & \text { on } \Sigma\end{cases}
$$

Remark: The formulation of $(\tilde{\mathcal{P}})$ corresponds to a convection-diffusion problem. However, setting $\tilde{\mathbf{v}}=\mathbf{0}$, we obtain the generic formulation of a diffusion problem.

The fictitious domain is the unit square $\Omega=] 0,1[\times] 0,1[$ (see Figure $4(\mathrm{~b})$ ).

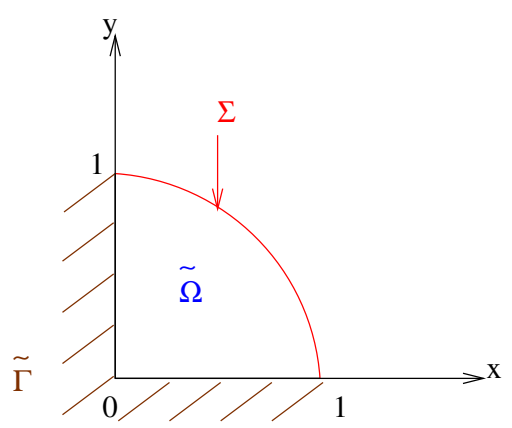

(a) Original domain $\tilde{\Omega}$

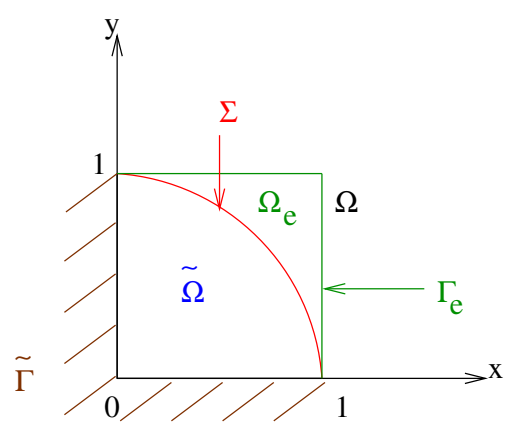

(b) Fictitious domain $\Omega$

Figure 4. Immersion of the unit disk in the unit square

The domain $\Omega$ is meshed uniformly with square cells $K$ with a grid step varying from $h=\frac{1}{4}$ to $h=\frac{1}{256}$. This defines the spread interface $\omega_{h, \Sigma}$ (see Figure 5). The problems $(\mathcal{P})$ solved in $\Omega$ are the ones defined in sections 2 and section 3 with symmetry conditions on $\tilde{\Gamma}$. 


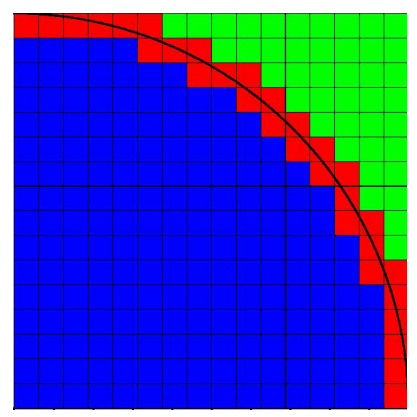

Figure 5. Discretization of $\Omega$ and definition of the spread interface $\omega_{h, \Sigma}$.

\subsection{Numerical methods}

\subsubsection{Approximation spaces and numerical solver}

The numerical resolution is performed using the $\mathcal{Q}_{1}$ finite element method (e.g. [39,27]). In this case, the usual simplicial Lagrange finite element space associated to the mesh $\mathcal{T}_{h}$ of $\Omega$ can be written as:

$$
V_{h}=\left\{v_{h} \in C^{0}(\bar{\Omega}) ;\left.v_{h}\right|_{K} \in \mathcal{Q}_{1}, \forall K \in \mathcal{T}_{h}\right\} \subset H^{1}(\Omega),
$$

where $\left\{\begin{array}{l}K \text { is an element of } \mathcal{T}_{h}, \\ \mathcal{Q}_{k} \text { stands for the space of polynomials of degree for each variable less than or equal to k, } \\ \frac{\text { Example: } \mathcal{Q}_{1}=\operatorname{span}\{1, x, y, x y\} \text { in } \mathbb{R}^{2}}{\text { The } \mathcal{Q}_{1} \text { discretization nodes are located on the vertices of the elements } K .}\end{array}\right.$ Within a $\mathcal{Q}_{1}$ finite element numerical approach, we set :

$$
\left\{\begin{array}{l}
\mathbf{a}_{h}, b_{h}, f_{h} \in \mathcal{Q}_{0}\left(\Omega_{h}\right) \\
u_{h}, \mathbf{v}_{h} \in \mathcal{Q}_{1}\left(\Omega_{h}\right)
\end{array}\right.
$$

where the subscript $h$ denotes the F.E. approximation of the original variable, and $u_{h}=u_{\eta, h}^{h}$.

For diffusion problems, when the matrix $\mathbf{a}$ is symmetric, the matrix of the linear system becomes symmetric positive definite. A conjugate gradient iterative algorithm [40] is used to solve the linear system. A diagonal preconditioner is used in order to improve the ill-conditioning due to the penalization coefficients. However, a more efficient preconditionner would be the inverse of the mass matrix.

For convection-diffusion problems, a conjugate gradient squared algorithm [41] is used to solve the linear system. In this case, a ILLU preconditioner is implemented . 


\subsubsection{Notations}

In order to evaluate the accuracy of the different variants presented in the previous sections, several error calculations are performed. As the analytic solution in the original domain of the test problems can be determined for coefficients of $(\tilde{\mathcal{P}})$ correctly chosen, the error $e_{h}$ is defined as the difference in $\underline{\tilde{\Omega}_{h}}$ between the analytic solution $\tilde{u}$ and the computational one $u_{h}$ :

$$
e_{h}=\tilde{u}-u_{h}
$$

A discrete $L^{2}{ }^{-}$norm is computed on the approximated original domain $\tilde{\Omega}_{h}$ :

$$
\forall \varphi_{h} \in \mathcal{Q}_{1}, \quad\left\|\varphi_{h}\right\|_{L^{2}\left(\tilde{\Omega}_{h}\right)}^{2}=\left(\sum_{K \in \mathcal{T}_{h}, K \subset \tilde{\Omega}_{h}}\left\|\varphi_{h}\right\|_{L^{2}(K)}^{2}\right)
$$

where $\left\|\varphi_{h}\right\|_{L^{2}(K)}^{2}$ is performed using a numerical integration exact on $\mathcal{Q}_{1}(K)$ :

$$
\int_{K} \varphi_{h}^{2} d x=\sum_{i=1}^{4} \frac{\operatorname{meas}(K)}{4} \varphi_{h}^{2}\left(x_{i}\right)+R\left(\varphi_{h}^{2}\right)
$$

(the subscript i denotes the vertex of the element $K$ ) with $R(\varphi) \equiv 0$ if $\varphi \in \mathcal{Q}_{1}$.

Here $R\left(\varphi_{h}^{2}\right) \neq 0$ because $\varphi_{h} \in \mathcal{Q}_{1} \Rightarrow \varphi_{h}^{2} \in \mathcal{Q}_{2}$. However the approximated coefficients of the fictitious problem $(\mathcal{P})$ are $\mathcal{Q}_{0}$. In the numerical resolution, the integrals on $\tilde{\Omega}_{h}$ are then roughly approximated. Thus, a $\mathcal{Q}_{1}$ numerical integration is sufficient to compute the discrete $L^{2}$ error norm. Hence, the solution $u_{h}$ does not need to be reconstructed in the $\mathcal{Q}_{1}$ base.

Finally,

$$
\left\|\varphi_{h}\right\|_{L^{2}\left(\tilde{\Omega}_{h}\right)}^{2} \simeq \sum_{K \in \mathcal{T}_{h}, K \subset \tilde{\Omega}_{h}} \sum_{i=1}^{4} \frac{\operatorname{meas}(K)}{4} \varphi_{h}^{2}\left(x_{i}\right)
$$

The discrete $L^{2}$ norm of the error $e_{h}$ in the approximated original domain $\tilde{\Omega}_{h}$ will be called absolute discrete $L^{2}$ error norm :

$$
\left\|\left.e_{h}\right|_{L^{2}\left(\tilde{\Omega}_{h}\right)}=\right\| \tilde{u}-u_{h} \|_{L^{2}\left(\tilde{\Omega}_{h}\right)}
$$

while the relative discrete $L^{2}$ error norm will be the ratio of the absolute discrete $L^{2}$ error norm to the discrete $L^{2}$ norm of the analytic solution $\tilde{u}$ :

$$
\frac{\left\|e_{h}\right\|_{L^{2}\left(\tilde{\Omega}_{h}\right)}}{\|\tilde{u}\|_{L^{2}\left(\tilde{\Omega}_{h}\right)}}=\frac{\left\|\tilde{u}-u_{h}\right\|_{L^{2}\left(\tilde{\Omega}_{h}\right)}}{\|\tilde{u}\|_{L^{2}\left(\tilde{\Omega}_{h}\right)}}
$$

The error can be divided into three parts: a modelling error, an interface discretization error and a numerical scheme error. If $u_{\eta}$ denotes the solution 
of the continuous fictitious problem (depending on $\eta$ due either to the penalization in the Dirichlet case or the vanishing of the external flux in the Robin case), we have:

$$
\left\|\tilde{u}-u_{h}\right\| \leqslant \underbrace{\left\|\tilde{u}-u_{\eta}\right\|}_{\text {modelling error }}+\underbrace{\underbrace{\left\|u_{\eta}-u_{\eta}^{h}\right\|}_{\text {interface discretization error }}+\underbrace{\left\|u_{\eta}^{h}-u_{h}\right\|}_{\text {numerical scheme error }}}_{\text {discretization error }}
$$

In order to study the behavior of the modelling error, the discretization error has to be negligible compared to the modelling one. The discretization step has to be very small. To study the discretization error, a small penalization parameter $\eta$ is chosen.

\subsubsection{Local adaptive mesh refinement}

After selecting the best approach (in term of error value), a local adaptive mesh refinement is performed. As the immersed interface $\Sigma$ is coarsely approximated by the spread interface $\omega_{h, \Sigma}$, the refinement area is chosen around the spread interface in order to improve the accuracy of the solution. A three-grid LDC algorithm (two refinement levels) is applied on each initial mesh. At each level, the local refinement zone is composed by all the elements of the spread interface $\omega_{h, \Sigma}$ and their neighbors. This choice makes it possible to correct the values of all the nodes located in the spread interface (see the LDC algorithm Section 4). If $G_{l}$ is the initial coarse grid, the local nested sub-grids $G_{l+p}$ are such that $h_{l+p}=\frac{h_{l}}{2^{p}}$ (see Figure 6$)$.

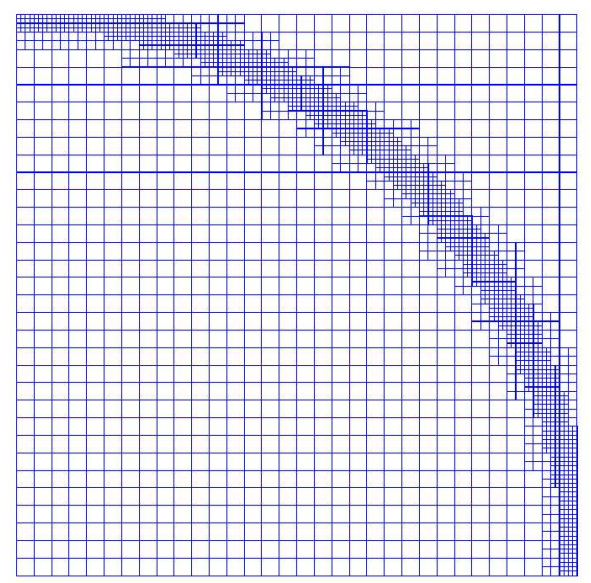

Figure 6. Example of a three-grid composite mesh

When the local adaptive mesh refinement is computed, it can be interesting to compute a composite $L^{2}$ error norm instead of the "coarse" discrete $L^{2}$ error norm on the original domain. On the initial coarse domain, this composite $L^{2}$ 
norm enables us to take into account the approximated solutions obtained on the local fine nodes.

The composite $L^{2}$ norm is calculated on the initial domain by the same way as the "coarse" discrete $L^{2}$ norm. The only difference between these two norms stands in the $L^{2}$ norm evaluation of the refined elements. For the evaluation of the composite $L^{2}$ norm, the $L^{2}$ error norm on the refined elements is estimated by the sum of the discrete $L^{2}$ error norms on each local fine element (of the fine grid generated from the current element) included in this coarse element. This algorithm is recursively applied to each element until the local finest level is reached.

On each nested grid, the iterative solvers have to deal with a relatively small number of degrees of freedom only. Thus, the implementation of this refinement method is relatively cheap.

\subsection{Results}

\subsubsection{Diffusion problems}

\subsubsection{Dirichlet case}

The problem solved in the original domain $\tilde{\Omega}$ is:

$$
(\tilde{\mathcal{P}}) \quad\left\{\begin{aligned}
-\triangle \tilde{u} & =4 \text { in } \tilde{\Omega} \\
\frac{\partial \tilde{u}}{\partial n} & =0 \text { on } \tilde{\Gamma} \\
\tilde{u} & =0 \text { on } \Sigma
\end{aligned}\right.
$$

With the generic formulation of the diffusion problem $(\tilde{\mathcal{P}})$ (see section 2.2)

$$
\tilde{a} \equiv 1(\tilde{\mathbf{a}} \equiv \mathbf{I d}), \tilde{f} \equiv 4 \text { and } \tilde{u}=u_{D}=0 \text { on } \Sigma(\text { Dirichlet B.C. })
$$

The analytic solution of this problem is:

$$
\tilde{u}=1-r^{2} \quad \text { in } \tilde{\Omega} \quad \text { where } r=\sqrt{x^{2}+y^{2}}
$$

This problem is computed on the fictitious square domain using the two approaches described in Section 2.3.1.

First, the asymptotic behavior of the solution is investigated with respect to the penalization parameter $\eta$. Thus, the modelling error can be estimated. In order to have a discretization error as negligible as possible, a $256 \times 256$ mesh is used. The results obtained for the exterior penalization (second approach) are presented.

In Figure 7, it can be observed that the $L^{2}$-norm of the modelling error varies 


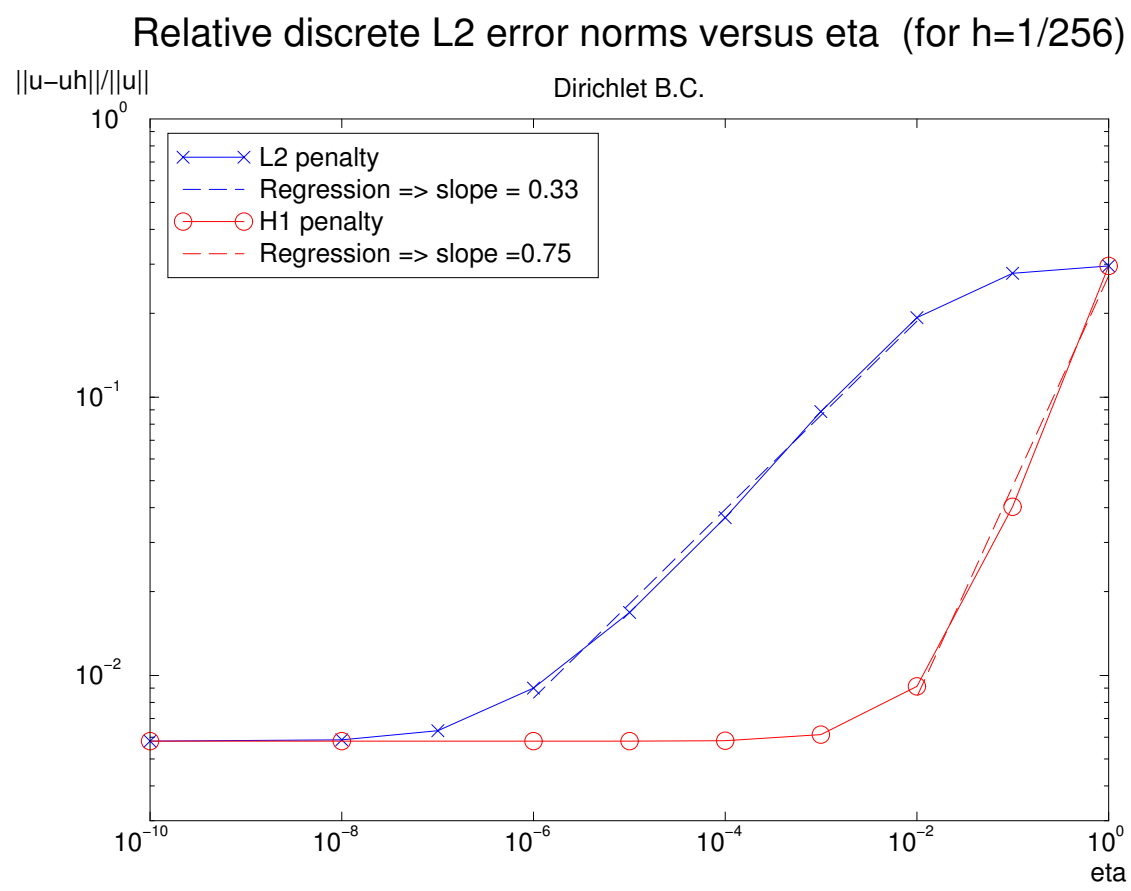

Figure 7. Modelling error for a Dirichlet diffusion problem with an exterior penalization

with respect to the penalization parameter $\eta$ like $\mathcal{O}\left(\eta^{\frac{1}{3}}\right)$ for the $L^{2}$ penalty, and like $\mathcal{O}\left(\eta^{\frac{3}{4}}\right)$ for the $H^{1}$ penalty. In [30], Angot theoretically estimates the $H^{1}$ norm of the modelling error with respect to $\eta$. These estimations are in $\mathcal{O}\left(\eta^{\frac{1}{4}}\right)$ for the $L^{2}$ penalty and in $\mathcal{O}(\eta)$ for the $H^{1}$ penalty. As the error estimates of the $L^{2}$-norm should be smaller, the asymptotic behavior of the modelling error obtained with the $H^{1}$ penalty is in contradiction with the error estimates of [30] in our approach. This can be explained by the fact that since the $H^{1}$ penalty modelling error converges rapidly, only a few points are used to estimate the asymptotic behavior of the modelling error.

The stagnation observed in Figure 7 corresponds to the discretization error. For small enough $\eta$, the modelling error becomes negligible compared to the discretization one, hence the error on the solution tends to the discretization error.

The following computations are performed with a $H^{1}$-penalty method setting $\eta=10^{-12}$ in order to obtain a negligible modelling error compared to the discretization error.

The approximated solutions obtained with the two Dirichlet approaches and the analytic one are represented in Figure 8. It can be seen that the penalty coefficient makes it possible to correctly impose $u_{h} \simeq u_{D}=0$ by penalizing either the spread interface (first approach) or the exterior domain (second approach). The exterior penalization seems to lead to a solution that is closer to the analytic solution than the solution obtained using the spread interface penalization. However, the accuracy of the different penalization methods 


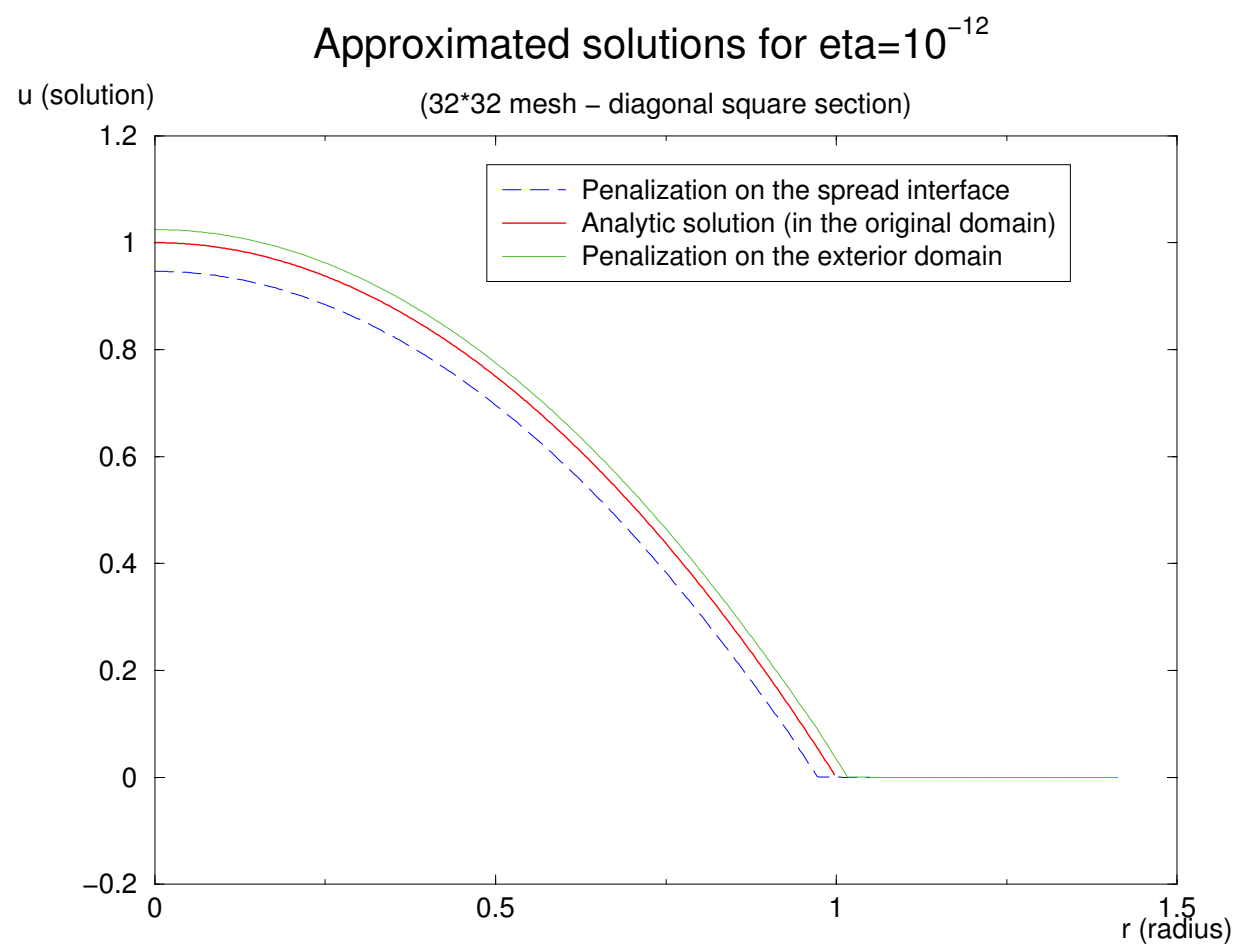

Figure 8. Approximated solutions in the Dirichlet case for a diffusion problem

seems to strictly depend on the geometry of the original domain $\tilde{\Omega}$. Performing the Finite Element Method, the reaction coefficient is computed by element $\left(b_{h} \in \mathcal{Q}_{0}\right)$. All the nodes belonging to a penalized element are then penalized. The spread interface penalization induces the penalization at $u_{D}$ of all the spread interface nodes. Hence, interior nodes of the original domain are penalized. The exterior penalization imposes $u_{h} \simeq u_{D}$ on the whole exterior domain and then on the exterior nodes of the spread interface. With an original geometry of quarter of a disk, interior penalized nodes are globally farther from the immersed interface $\Sigma$ than the exterior nodes of the spread interface (see Figure 5).

For each approach, the relative discrete $L^{2}$ error norms with respect to the discretization step $h$ are reported in Figure 9.

As $\left|\operatorname{meas}\left(\tilde{\Omega}_{h}\right)-\operatorname{meas}(\tilde{\Omega})\right|=\mathcal{O}(h)$, our fictitious domain approach is expected to be a first-order method. Figure 9 confirms that the two Dirichlet approaches are first-order methods. The interface discretization error leads to a "global" discretization error in $\mathcal{O}(h)$ even if the numerical scheme error is in $\mathcal{O}\left(h^{2}\right)$ for the $L^{2}$-norm (see [39]).

As illustrated in Figure 10, the main differences between the approximated solution and the analytic one are located around the spread interface. An adaptive mesh refinement is performed in this zone. At each level, an $H^{1}$ exterior penalization is performed. The three-grid LDC algorithm converges within three $\mathrm{V}$-cycles (see Figure 11). The relative discrete $L^{2}$ error norms on 


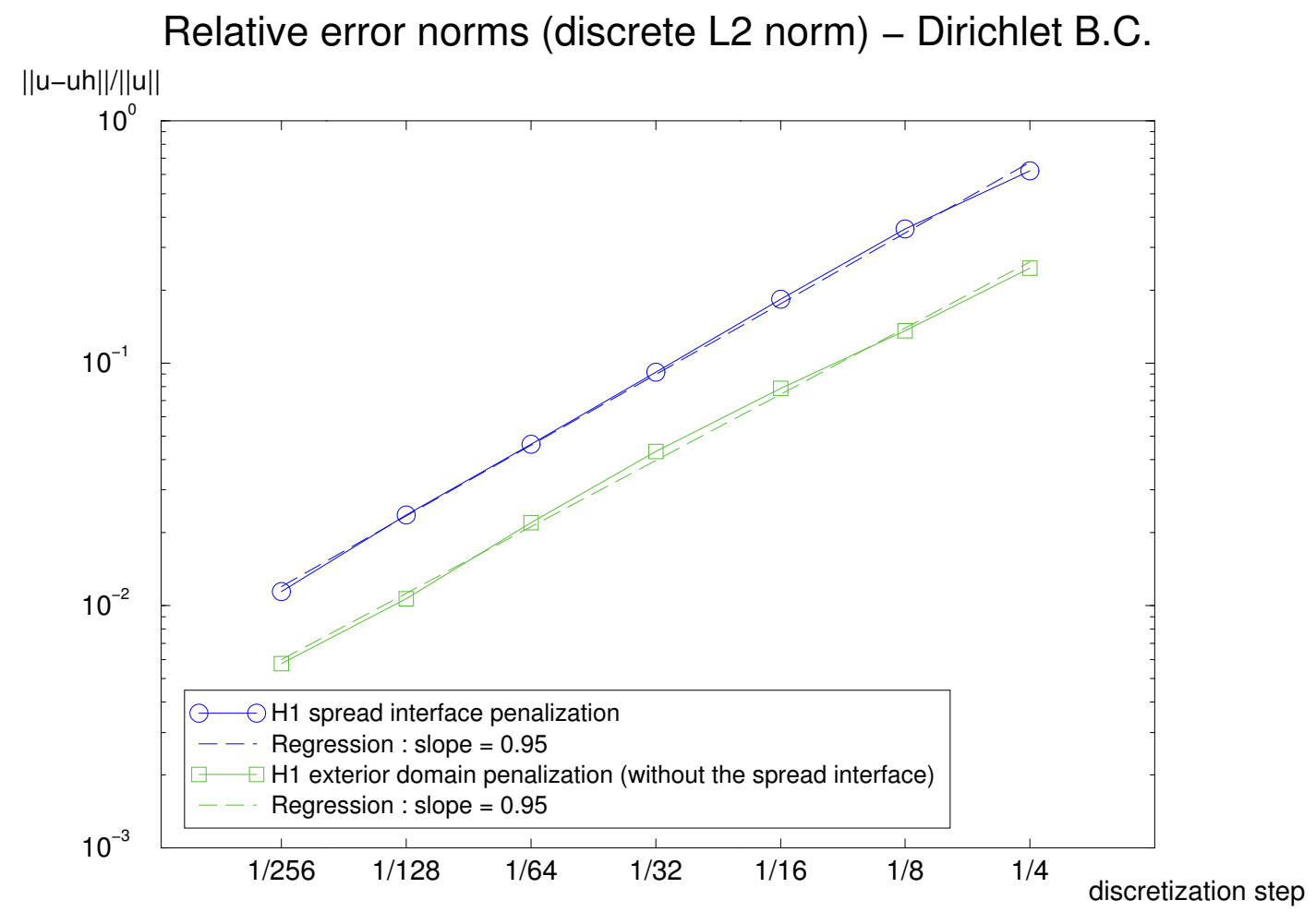

Figure 9. Discretization errors for a Dirichlet diffusion problem

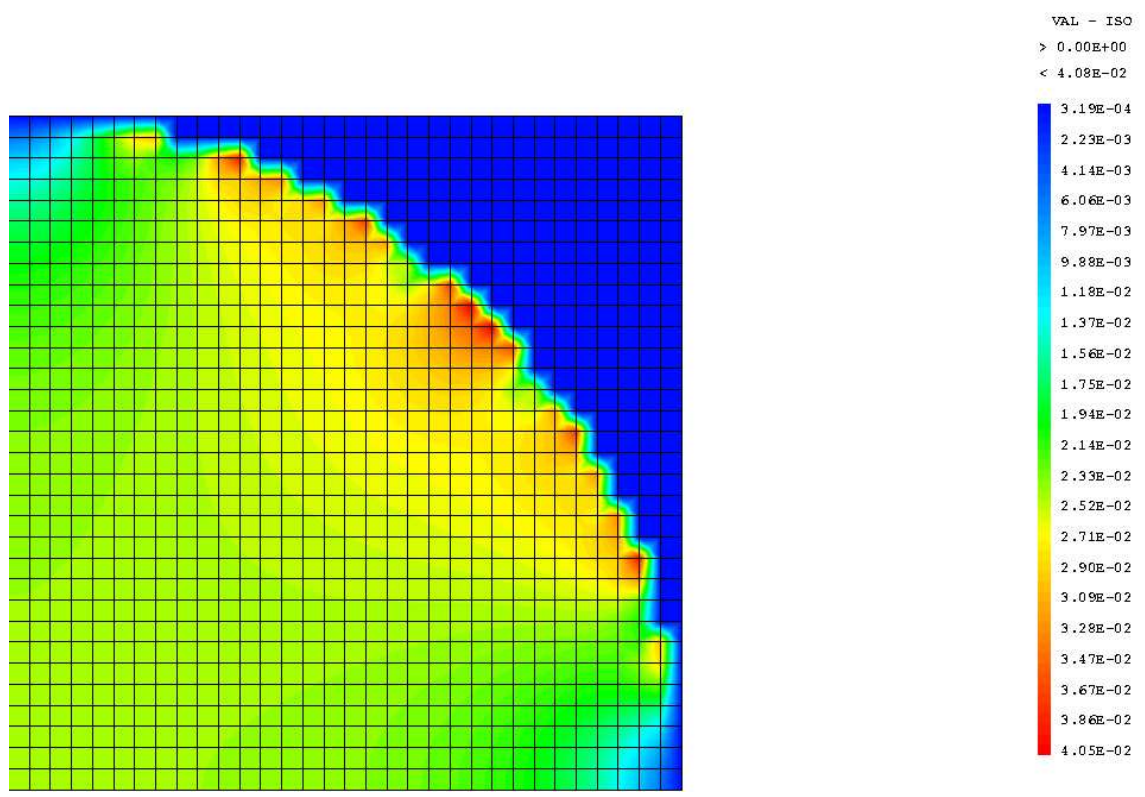

Figure 10. Error distribution for the exterior penalization - Dirichlet diffusion problem - 32x32 mesh

the fine levels have been computed extending by 0 the error in the part of the original domain $\tilde{\Omega}_{h}$ with an empty intersection with the refinement zone. Moreover, on each level, the absolute discrete $L^{2}$ error norm is divided by the 
same coarse absolute discrete $L^{2}$ norm of the analytic solution.

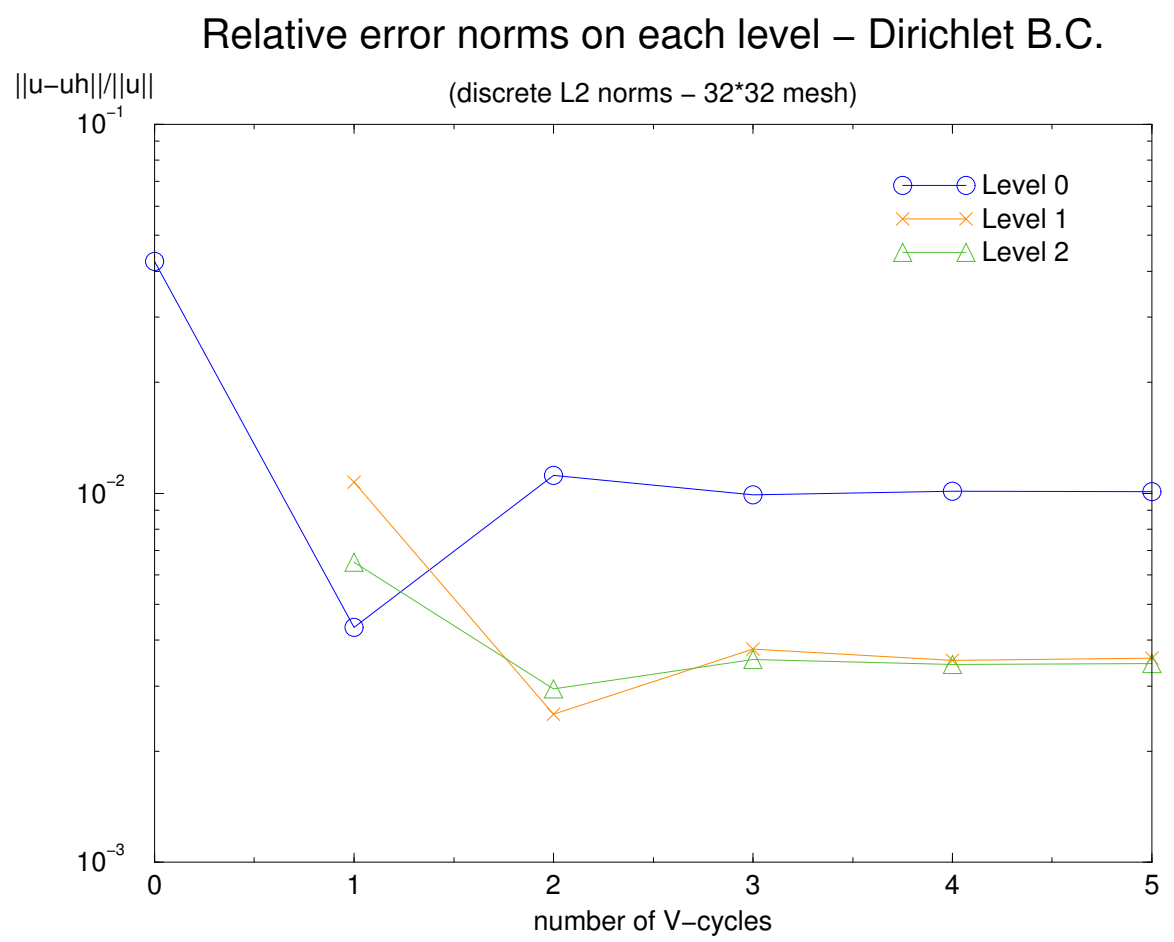

Figure 11. Representation of the relative discrete $L^{2}$ error norms on each refinement level - Dirichlet diffusion problem

As shown in Figure 12, with a local refinement around the immersed interface $\Sigma$, the error on the initial coarse grid is similar to the error obtained without refinement on a mesh with a discretization step equal to the local finest grid's one. It confirms that the most important error is located near the immersed interface. Moreover, for fine mesh steps, the composite $L^{2}$ error norm and the relative $L^{2}$ error norm on the initial coarse domain are similar. Figure 12 enables us to conclude that the results obtained with refinement vary like $\mathcal{O}\left(h_{f}\right)$ where $h_{f}$ is the discretization step of the local finest refinement grid (last level of refinement). The combination of local adaptive mesh refinement with the spread interface fictitious domain method allows us to increase the precision of the solution even if the method remains first-order.

\subsubsection{Robin case}

The following problem is considered:

$$
(\tilde{\mathcal{P}}) \quad\left\{\begin{aligned}
-\triangle \tilde{u} & =16 r^{2} \quad \text { in } \tilde{\Omega} \\
\frac{\partial \tilde{u}}{\partial n} & =0 \quad \text { on } \tilde{\Gamma} \\
-\frac{\partial \tilde{u}}{\partial n} & =u+3 \text { on } \Sigma
\end{aligned}\right.
$$


Relative L2 error norms - F.D.M. + A.M.R. -Dirichlet B.C.

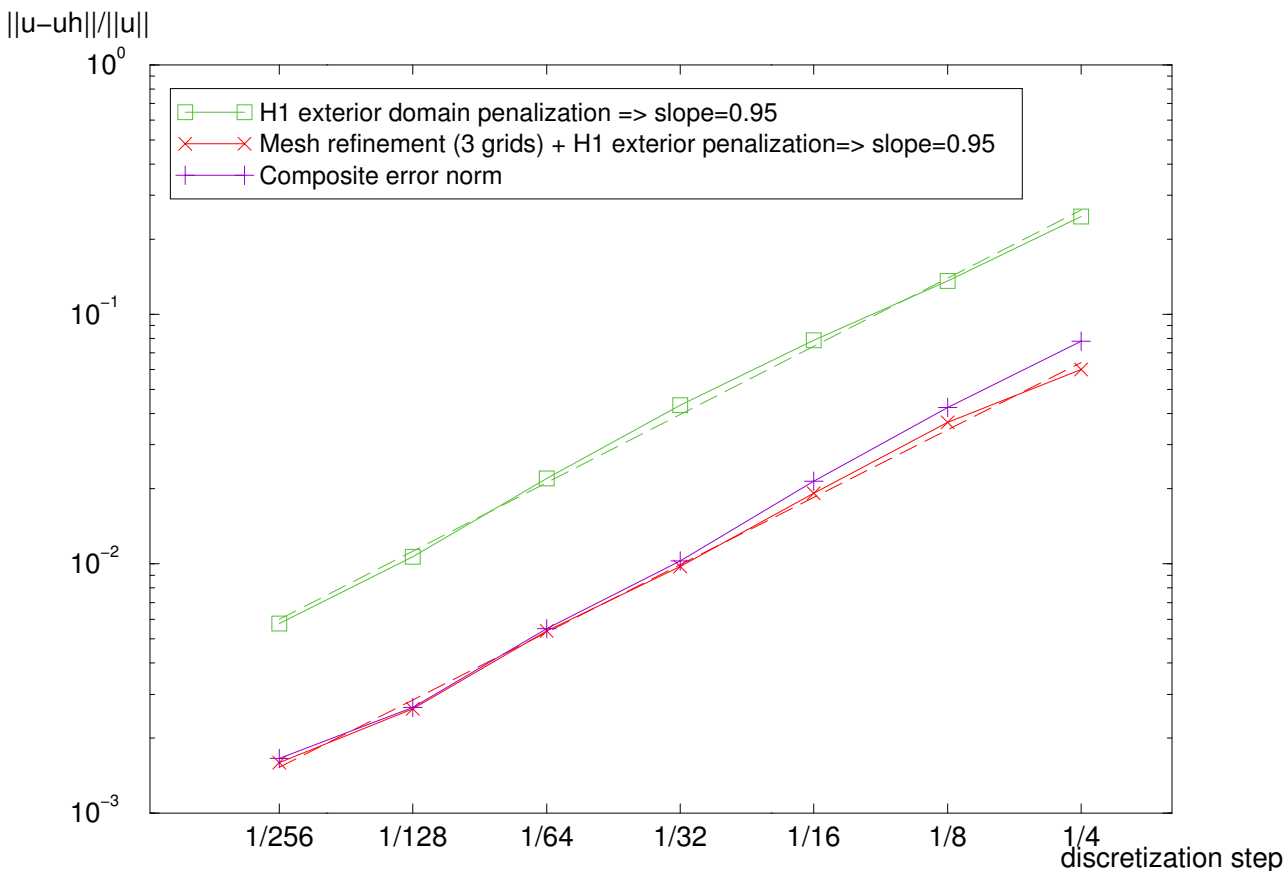

Figure 12. Discretization errors with or without refinement - Exterior penalization Dirichlet diffusion problem

Identifying with the generic formulation $(\tilde{\mathcal{P}})$, we get:

$$
\tilde{a} \equiv 1(\tilde{\mathbf{a}} \equiv \mathbf{I d}), \tilde{f}=16 r^{2}, \alpha_{R}=1 \text { and } g_{R}=3 \text { (Robin B.C.) }
$$

The solution of this problem is:

$$
\tilde{u}=2-r^{4} \quad \text { in } \tilde{\Omega}
$$

The behavior of the three approaches presented in Section 2.3.2 are investigated. We chose $\eta=10^{-12}$ to make the external flux vanish. The modelling error is then negligible.

In Figure 13, all these approaches are approximately first-order methods up to a $128 \times 128$ mesh. For a $256 \times 256$ mesh, error stagnation seems appear for the variants involving a global approximation of the characteristic parameter $\epsilon$. The method using a piecewise linear approximation of the interface leads to slightly smaller errors.

Local adaptive mesh refinement is performed on the methods involving either global approximations of epsilon (constant case $\epsilon=\frac{\operatorname{meas}\left(\omega_{h, \Sigma}\right)}{\operatorname{meas}(\Sigma)}$ and volume weighted case $\left.\epsilon_{K}=\frac{\sum_{K}\left[\tau_{K} \cdot \operatorname{meas}(K)\right]}{\tau_{K} \cdot \operatorname{meas}(\Sigma)}\right)$ or a local approximation of 


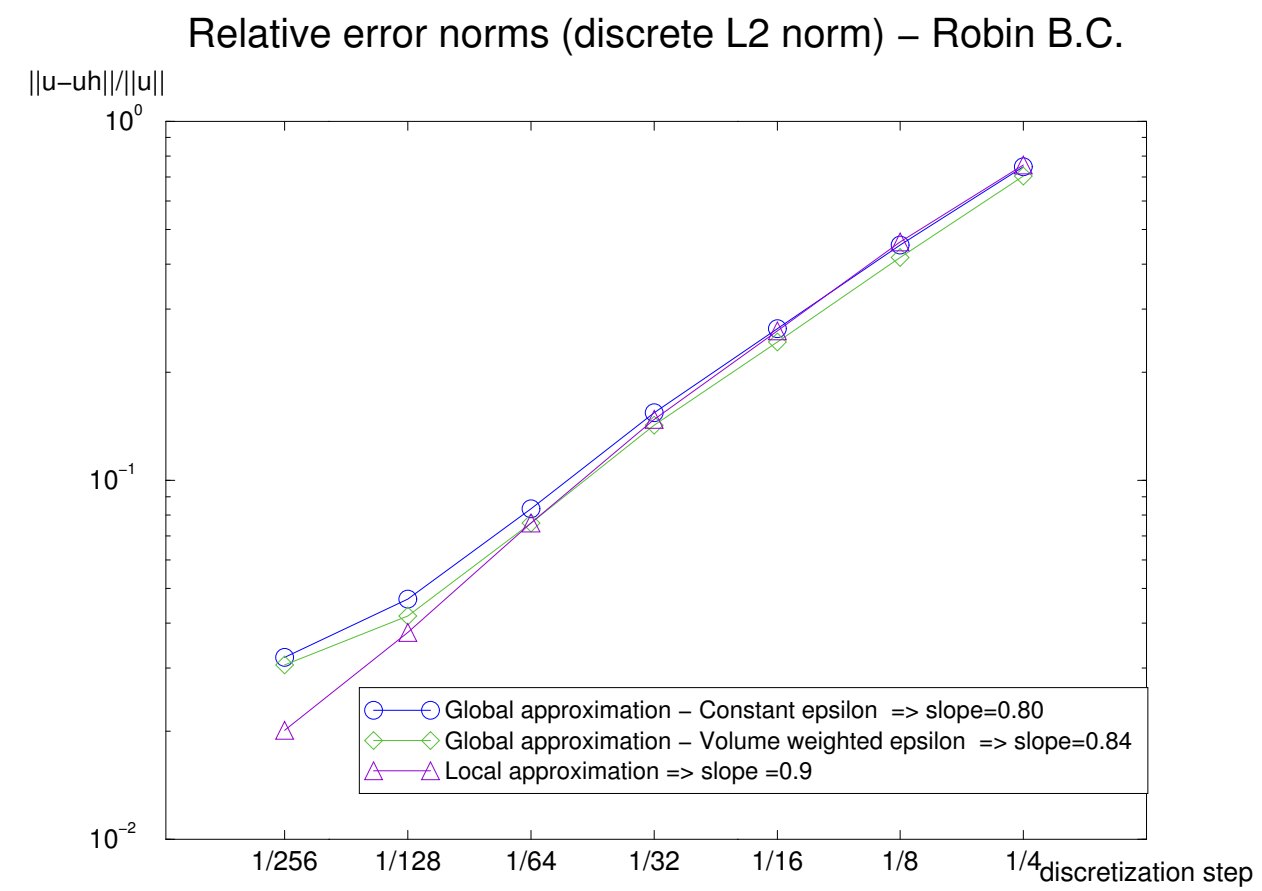

Figure 13. Discretization errors for a Robin diffusion problem

epsilon (linear approximation $\left.\epsilon_{K}=\frac{\operatorname{meas}(K)}{\operatorname{meas}\left(\Sigma_{l, K}\right)}\right)$. As in the Dirichlet case, a three-grid LDC algorithm is computed, which converges by three V-cycles.

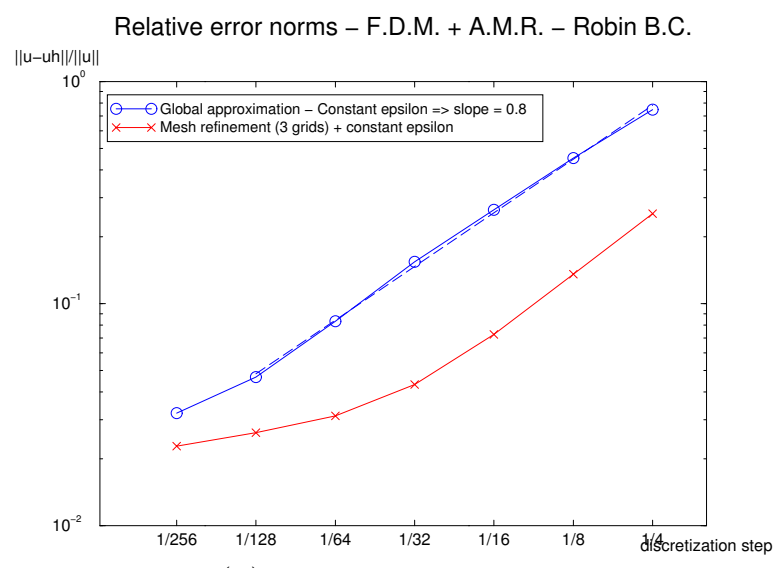

(a) Constant epsilon

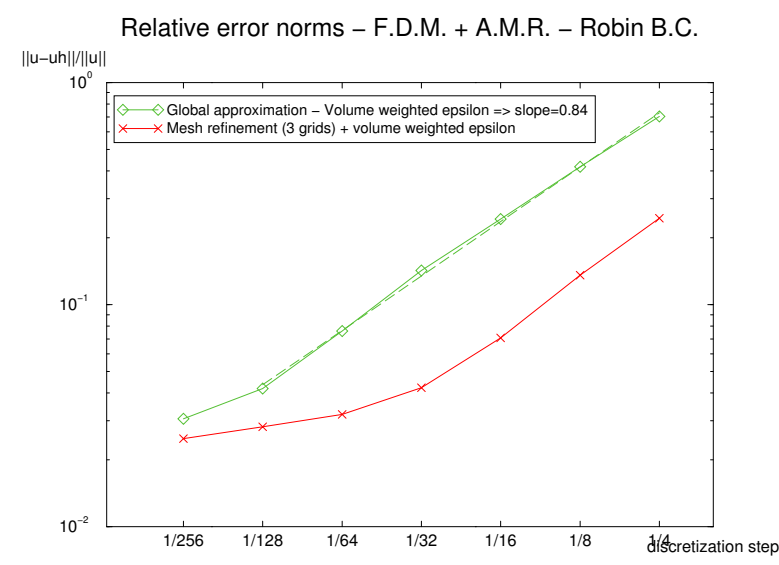

(b) Volume weighted epsilon

Figure 14. Discretization errors with or without refinement - Global approximations of $\epsilon$ - Robin diffusion problem

The results obtained with the local mesh refinement (see Figures 14 and 15) confirm the first conclusions drawn from Figure 13. The methods with global approximations of $\epsilon$ do not remain the first-order accuracy for fine meshes. Indeed, error stagnation is observed in Figure 14. Such stagnation is due to the global estimation of $\epsilon$ (equation (6)) since no stagnation appears with a local approximation of $\epsilon$ (see Figure 15). A local correction enables us to have a discretization error in $\mathcal{O}\left(h_{f}\right)$ with $h_{f}$ the discretization step of the local finest grid. 


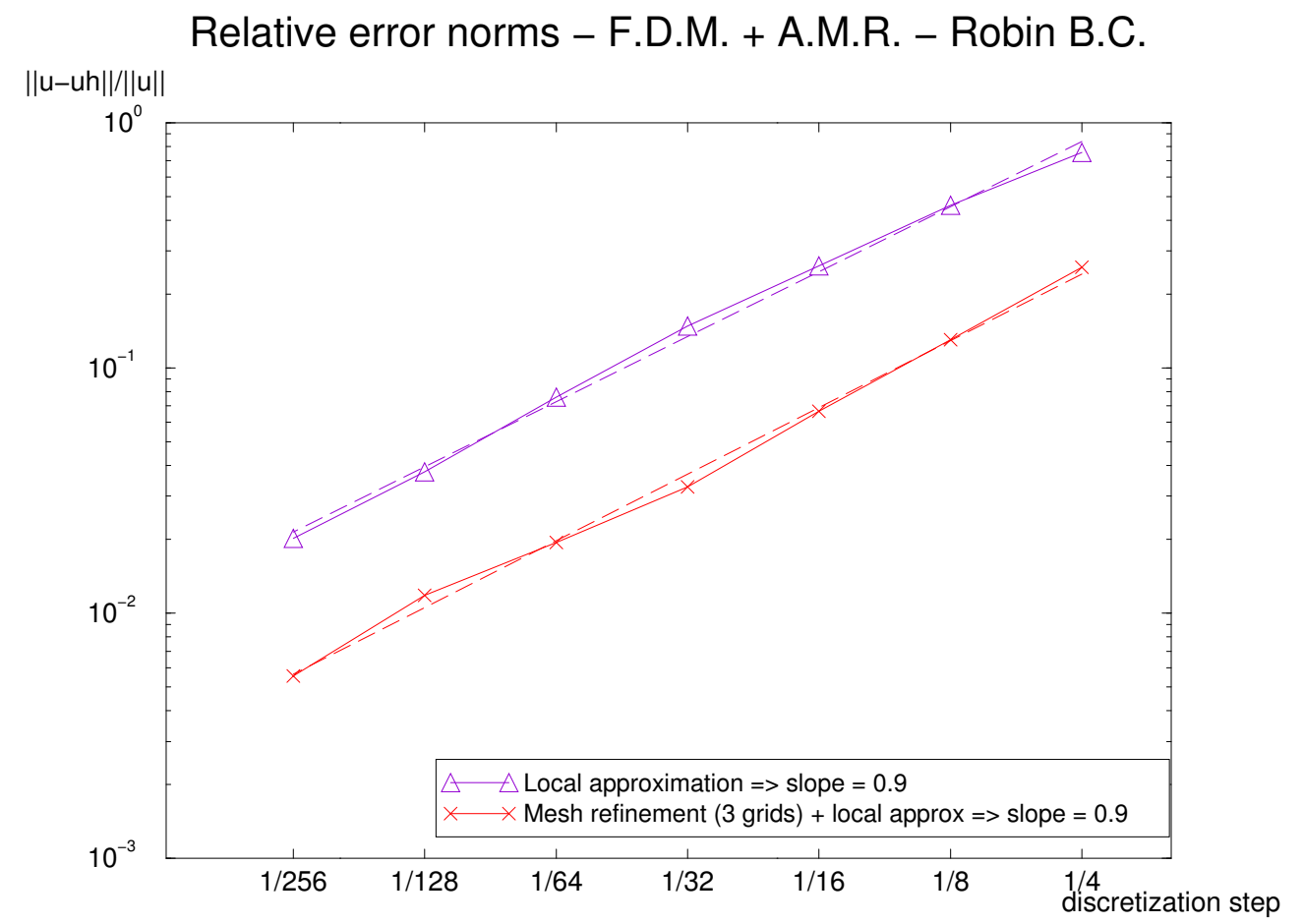

Figure 15. Discretization errors with or without refinement - Local approximation of $\epsilon$ - Robin problem

\subsubsection{Convection-diffusion problems}

\subsubsection{Dirichlet problem}

The Dirichlet problem is considered as follows:

$$
(\tilde{\mathcal{P}})\left\{\begin{aligned}
-\Delta \tilde{u}+\operatorname{div}(\tilde{\mathbf{v}} \tilde{u}) & =4 \text { in } \tilde{\Omega} \\
\frac{\partial \tilde{u}}{\partial n} & =0 \text { on } \tilde{\Gamma} \\
\tilde{u}=u_{D} & =0 \text { on } \Sigma
\end{aligned}\right.
$$

with $\tilde{\mathbf{v}}=\frac{r}{2} \mathbf{e}_{\mathbf{r}}$ where $r=\sqrt{x^{2}+y^{2}}$ and $\mathbf{e}_{\mathbf{r}}$ is the radial unit vector.

The analytic solution of this problem is:

$$
\tilde{u}=4\left(1-\exp \left(\frac{r^{2}-1}{4}\right)\right) \quad \text { in } \tilde{\Omega}
$$

The associated fictitious problem $(\mathcal{P})$ (see section 3.1) with a spread interface approach is solved in $\Omega$ with the two Dirichlet embedded B.C. methods: the spread interface penalization and the exterior penalization (see Table 2). The following results have been performed with $\eta=10^{-12}$ to obtain a negli- 
gible modelling error compared to the discretization error.

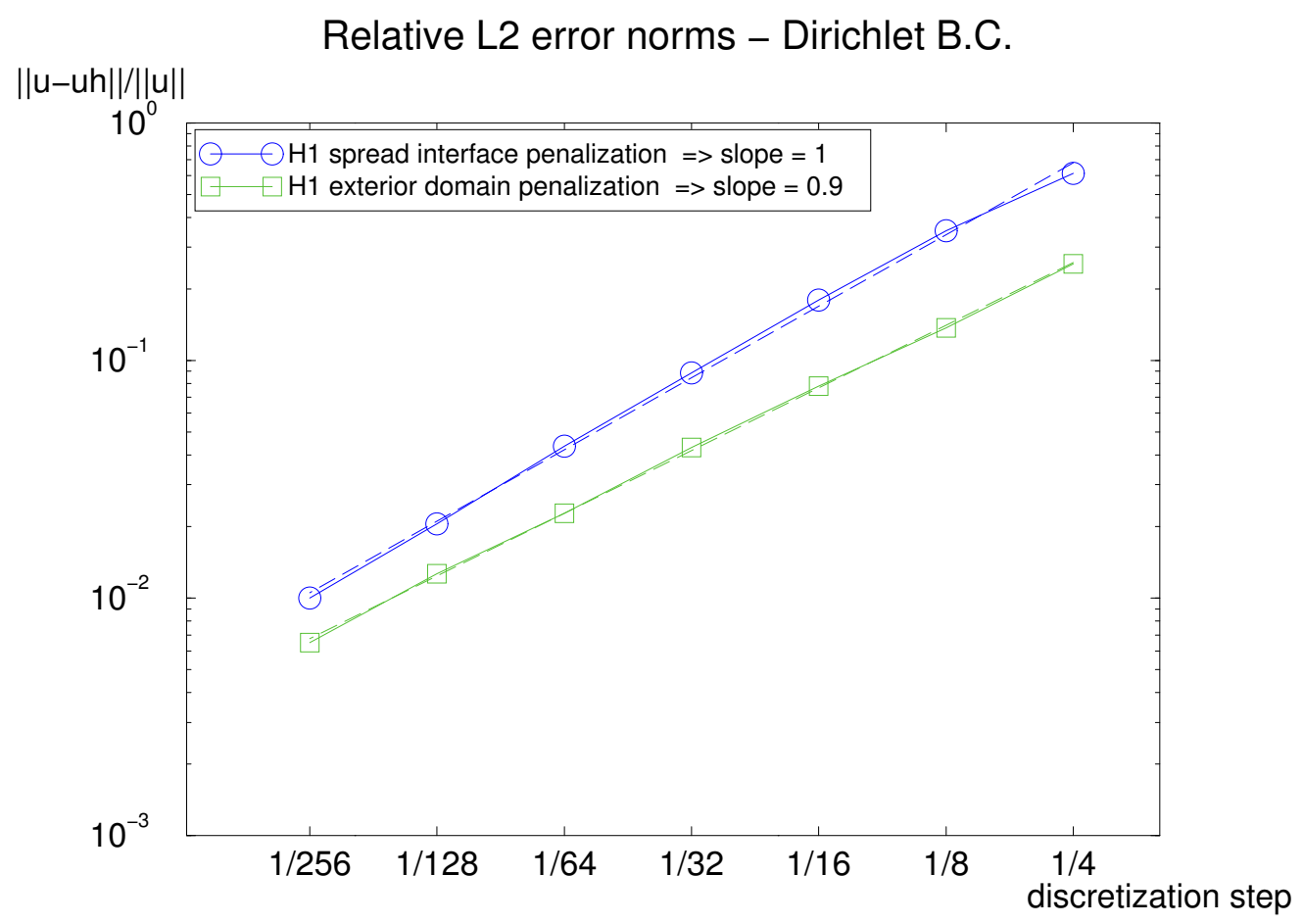

Figure 16. Discretization errors for a Dirichlet convection-diffusion problem

Figure 16 shows the convergence of the numerical errors (performed with the $H^{1}$ penalty) with respect to the mesh step $h$. As for a diffusion problem, since $\left|\operatorname{meas}\left(\tilde{\Omega}_{h}\right)-\operatorname{meas}(\tilde{\Omega})\right|=\mathcal{O}(h)$ all the Dirichlet embedded B.C. variants are of first-order accuracy for the $L^{2}$-norm.

An adaptive mesh refinement is performed around the immersed interface. On each local grid, a $\mathcal{Q}_{1}$ F.E. scheme with an $H^{1}$ exterior domain penalization method is computed. A three-grid LDC algorithm (two refinement levels) is applied on each original mesh. As for the diffusion case, this algorithm converges by three $\mathrm{V}$-cycles.

As shown in Figure 17, once again the spread interface method combined with a L.D.C. algorithm is a first-order method with respect to the discretization step of the local finest refinement grid. 


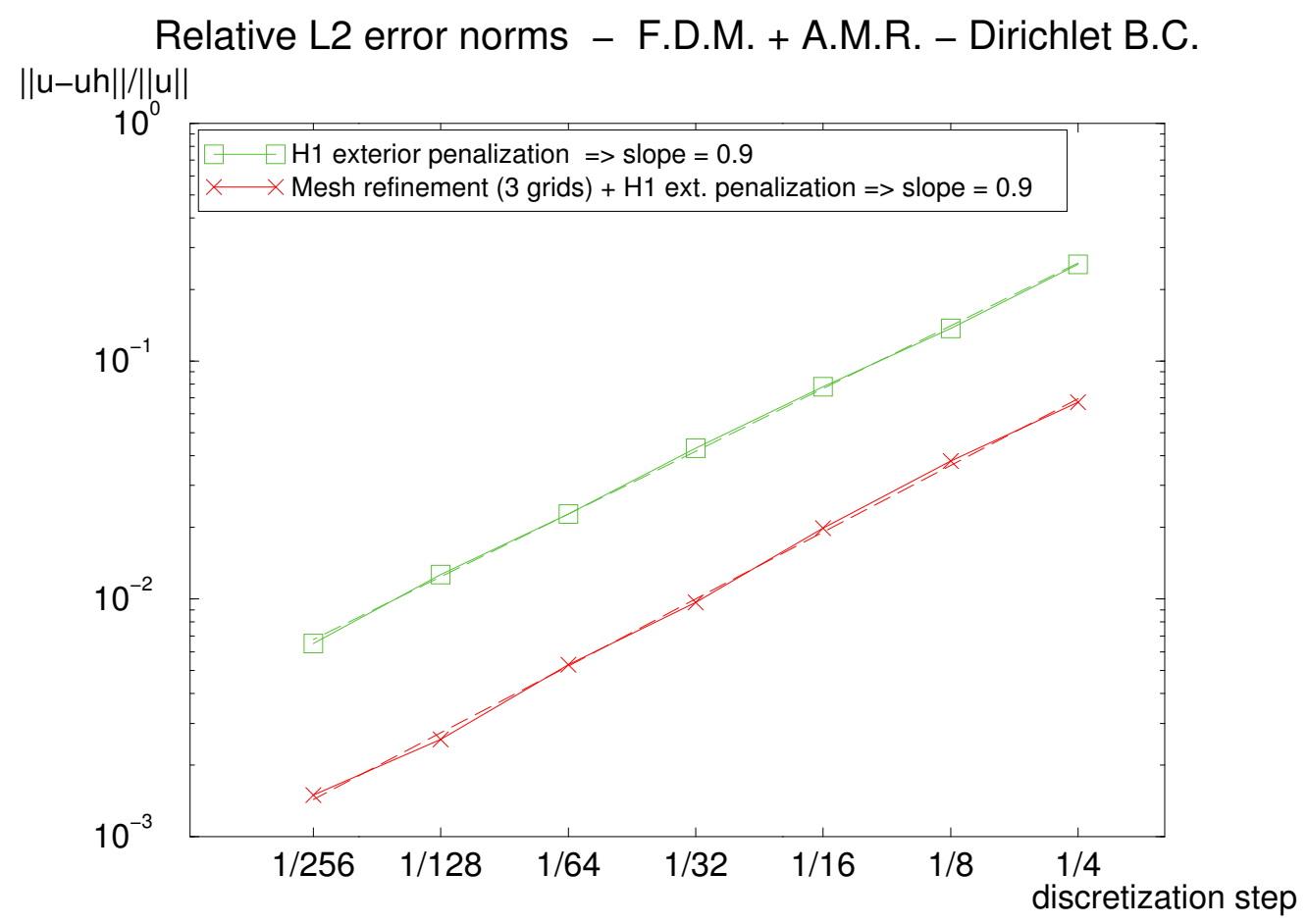

Figure 17. Discretization errors with or without refinement - Exterior penalization Dirichlet convection-diffusion problem

\subsubsection{Robin problem}

The following Robin problem is considered:

$$
(\tilde{\mathcal{P}}) \quad\left\{\begin{aligned}
-\triangle \tilde{u}+\operatorname{div}(\tilde{\mathbf{v}} \tilde{u}) & =16 r^{2} \quad \text { in } \tilde{\Omega} \\
\frac{\partial \tilde{u}}{\partial n} & =0 \quad \text { on } \tilde{\Gamma} \\
\frac{\partial \tilde{u}}{\partial n} & =\tilde{u}+3 \text { on } \Sigma\left(\alpha_{R}=1, g_{R}=3\right)
\end{aligned}\right.
$$

with $\tilde{\mathbf{v}}=2 r^{3} \mathbf{e}_{\mathbf{r}}$.

The analytic solution of this problem is:

$$
\tilde{u}=2-\frac{5}{3} \exp \left(\frac{r^{4}-1}{2}\right) \quad \text { in } \tilde{\Omega}
$$

The fictitious problem $(\mathcal{P})$ is solved in $\Omega$ with an embedded Robin B.C. in the spread interface (see Table 2). The results obtained with the three approximations of the characteristic parameter $\epsilon$ discussed in section 2.3.2 are reported in Figure 18.

A global approximation of the characteristic parameter $\epsilon$ (see Eq. (7) and (8)) leads to an asymptotic stagnation of the error and then the first-order precision is lost. With a local correction (see Eq. (10)), the asymptotically 


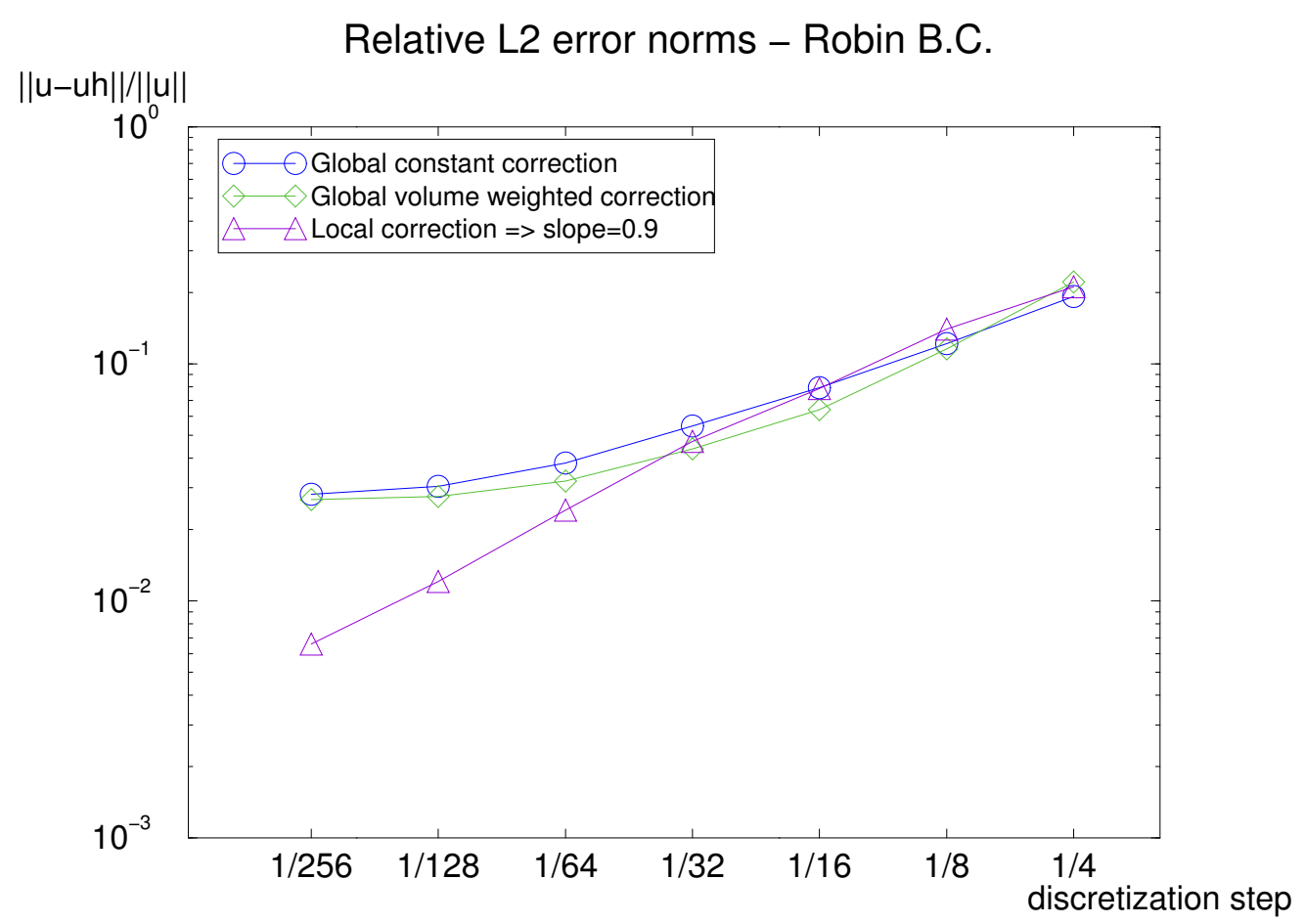

Figure 18. Discretization errors for a Robin convection-diffusion problem

first-order accuracy is then yielded for the $L^{2}$-norm error. Compared to diffusion problems, the asymptotic stagnation of the variants involving a global $\epsilon$ appears earlier, from the $32 \times 32$ mesh. For Robin problems, a local correction is thus required to keep the first-order method.

A local A.M.R. algorithm is performed on the method involving a local epsilon (Eq. (10)). As in the Dirichlet case, we compute a three-grid LDC algorithm, which converges by three $\mathrm{V}$-cycles.

Figure 19 illustrates that the combination of the spread interface fictitious domain method with an A.M.R. method leads to a discretization error in $\mathcal{O}\left(h_{f}\right)$ with $h_{f}$ the mesh step of the local finest grid.

\section{Conclusion}

The numerical results presented in the last section prove the efficiency of the fictitious domain method with a spread interface introduced in this article. This method enables us to handle all the usual B.C.. Moreover, the main advantage of this method is its low cost. The numerical resolution is computed on a single Cartesian mesh of the fictitious domain, without locally modifying the numerical scheme. No surface mesh (on the immersed interface) is required. Even if this is a first-order method, the combination with an adaptive multi- 


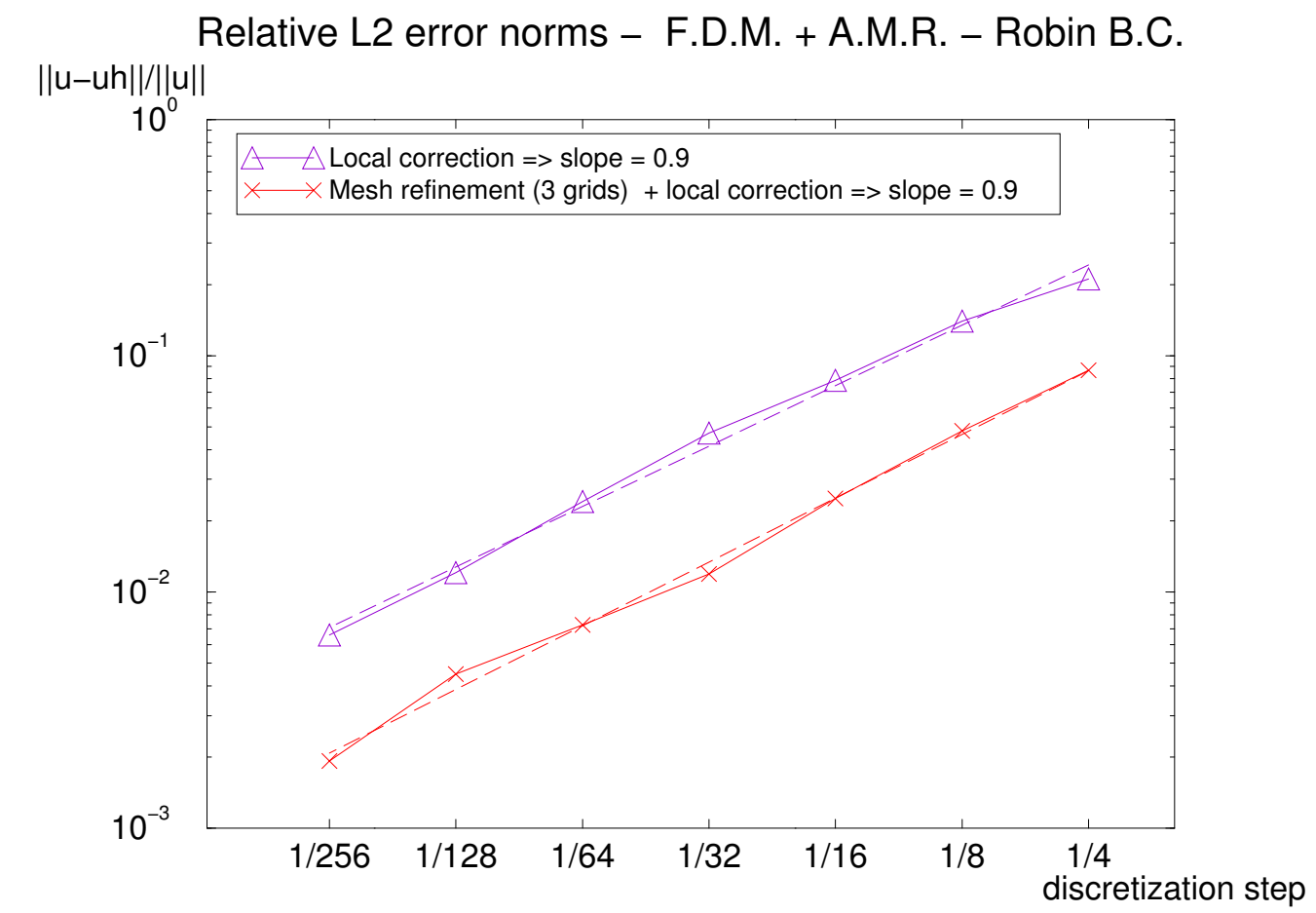

Figure 19. Discretization errors with and without refinement - Local approximation of $\epsilon$ - Robin convection-diffusion problem

level local mesh refinement solver yields an error on the coarse grid similar to the error obtained without refinement on a mesh with the local finest grid discretization step. The over-cost of the A.M.R. resolution is relatively cheap. Our fictitious domain method combined with a local adaptive mesh refinement is then accurate and relatively cheap in CPU time.

\section{References}

[1] V.K. Saul'ev. On the solution of some boundary value problems on high performance computers by fictitious domain method. Siberian Math. Journal, 4(4):912-925, 1963 (in Russian).

[2] G.I. Marchuk. Methods of Numerical Mathematics. Application of Math. 2, Springer-Verlag New York, 1982 (1rst ed. 1975).

[3] Ph. Angot, J.-P. Caltagirone, and K. Khadra. A comparison of locally adaptive multigrid methods: L.D.C., F.A.C. and F.I.C. NASA Conf. Publ. CP-3224, volume 1, pp.275-292, 1993.

[4] K. Khadra, Ph. Angot, S. Parneix, and J.-P. Caltagirone. Fictitious domain approach for numerical modelling of Navier-Stokes equations. Int. J. Numer. Meth. in Fluids, Vol. 34(8): 651-684, 2000. 
[5] R. Cortez and M. Minion. The blob projection method for immersed boundary problems. J. Comput. Phys., 161:428-463, 2000.

[6] P. McCorquodale, P. Colella, and H. Johansen. A Cartesian grid embedded boundary method for the heat equation on irregular domains. J. Comput. Phys., 173:620-635, 2001.

[7] T. Ye, R. Mittal, H.S. Udaykumar, and W. Shyy. An accurate Cartesian grid method for viscous incompressible flows with complex immersed boundaries. $J$. Comput. Phys., 156:209-240, 1999.

[8] R.J. Leveque and Z. Li. The immersed interface method for elliptic equations with discontinuous coefficients and singular sources. SIAM J. Numer. Anal., 31:1019-1044, 1994.

[9] A. Wiegman and K.P. Bube. The explicit-jump immersed interface method: finite difference methods for PDEs with piecewise smooth solutions. SIAM J. Numer. Anal., 37(3) pp. 827-862, 2000.

[10] R. Glowinski, T.-W. Pan, Jr.R. Wells, and X. Zhou. Wavelet and finite element solutions for the Neumann problem using fictitious domains. Journal Comput. Phys., Vol. 126(1),pp.40-51, 1996.

[11] Ph. Angot, Ch.-H. Bruneau, and P. Fabrie. A penalization method to take into account obstacle in incompressible viscous flows. Nümerische Mathematik, 81(4):497-520, 1999.

[12] Ph. Angot. Finite volume methods for non smooth solution of diffusion models; Application to imperfect contact problems. Numerical Methods ans Applications-Proceedings 4th Int. Conf. NMA'98, Sofia (Bulgaria) 19-23 August 1998, pp.621-629, World Scientific Publishing, 1999.

[13] E.J. Dean, Q.V. Dinh, R. Glowinski, J. He, T.W. Pan, and J. Periaux. Least squares/domain imbedding methods for Neumann problems: application to fluid dynamics. Domain Decomposition Methods for Partial Differential Equations, D. E. Keyes, T. F. Chan, G. Meurant, J. S. Scroggs, R. G. Voigt eds., SIAM, Philadelphia, pp. 451-475, 1992.

[14] R. Glowinski, T.-W. Pan, and J. Peraux. A fictitious domain method for Dirichlet problem and applications. Computer Methods in Applied Mechanics and Engineering 111 (3-4): 283-303, 1994.

[15] R. Glowinski and Y. Kuznetsov. On the solution of the Dirichlet problem for linear elliptic operators by a distributed Lagrange multiplier method. C.R. Acad. Sci. Paris, t.327, Seie I, pp. 693-698, 1998.

[16] J.-C. Latché. A fictitious degrees of freedom finite element method for free surface flows. Mathematical Modelling and Numerical Analysis, submitted (october 2003).

[17] Ph. Angot. A fictitious domain model for general embedded boundary conditions. C.R. Acad. Sci. Paris, Ser. Math., Vol. 341(11), pp 683-688, 2005. 
[18] Ph. Angot, H. Lomenède, and I. Ramière. A general fictitious domain method with non-conforming structured mesh. Finite Volume for Complex Applications IV, Problems and Perspectives, Ed. F. Benkhaldoun, D. Ouazar, S. Raghay - Hermes Science (2005), pp. 261-272. International Symposium on Finite Volumes IV (FVCA4). Marrakech, Marocco, 4-7 July, 2005.

[19] L.A. Rukhovets. A remark on the method of fictive domains. Differential Equations, 3,4, 1967 (in Russian).

[20] C.S. Peskin. Flow patterns around heart valves: A numerical method. $J$. Comput. Phys., Volume 10, Issue 2, Pages 252-271, 1972.

[21] C.S. Peskin. The immersed boundary method. Acta Numerica, Vol 11, pp 479-517, 2002.

[22] B. Maury. A Fat Boundary Method for the Poisson problem in a domain with holes. SIAM, J. of Sci. Comput., 16(3):319-339, 2001.

[23] M. Ismail. The Fat Boundary Method for the Numerical resolution of Elliptic Problems in Perforated Domains. Application to 3D Fluid Flows. PhD thesis, University Pierre and Marie Curie - Paris VI, France, 2004.

[24] L. Tomas. Shape Optimization and Fictitious Domains : analysis of new formulations and algorithm aspects. PhD thesis, Ecole Centrale de Lyon, France, 1997.

[25] V.D. Kopčenov. A method of fictitious domains for the second and third value problems. Trudy Mat. Inst. Steklov, Vol 131, pp.119-12\%, 1974 (in Russian).

[26] R. Dautray and J.L. Lions. Analyse mathématique et calcul numérique pour les sciences et les techniques, vol. 1-9. Masson, 1988.

[27] P.A. Raviart and J.M. Thomas. Introduction à l'analyse numérique des équations aux dérivées partielles. Masson, 1992.

[28] I. Ramière, M. Belliard, and Ph. Angot. On the simulation of Nuclear Power Plant Components using a fictitious domain approach. Proc. in the 11th International Topical Meeting on Nuclear Thermal-Hydraulics (NURETH-11), Avignon (France) 2-6 October, Paper 193, 2005.

[29] Ph. Angot. Contribution à l'étude des transferts thermiques dans des systèmes complexes; Application aux composants électroniques. Thèse de doctorat (Mécanique), Université de Bordeaux I, 1989.

[30] Ph. Angot. Analysis of singular perturbations on the Brinkman problem for fictitious domain models of viscous flows. $M^{2} A S$ Math. Meth. in the Appl. Sci., Vol. 22(16), pp. 1395-1412, 1999.

[31] F. Boyer and P. Fabrie. Eléments d'analyse pour l'étude de quelques modèles d'écoulements de fluides visqueux incompressibles. Mathématiques et Applications, Vol. 52, 2006.

[32] H. Brezis. Analyse fonctionnelle, Théorie et applications. Dunod, 2000. 
[33] I. Ramière, Ph. Angot, and M. Belliard. Fictitious domain methods to solve convection-diffusion problems with general boundary conditions. AIAA 2005-4709. 17th Computational Fluid Dynamics Conference - AIAA. Toronto, Canada, 6-9 June, 2005.

[34] W. Hackbusch. Multi-grid Methods and applications. Series in computer mathematics, Springer-Verlag, 1985.

[35] W. Hackbusch. Local Defect Correction Method and Domain Decomposition Techniques. Vol. 5 of Computing Suppl., pp 89-113, Springer-Verlag (Wien), 1984 .

[36] M. Grandotto and P. Obry. Calculs des écoulements diphasiques dans les échangeurs par une méthode aux éléments finis. Revue européenne des éléments finis. Volume 5, n¹/1996, pages 53 à 74, 1996.

[37] M. Grandotto, M. Bernard, J.P. Gaillard, J.L. Cheissoux, and E. De Langre. A 3D finite element analysis for solving two phase flow problems in PWR steam generators. In 7th International Conference on Finite Element Methods in Flow Problems, Huntsville, Alabama, USA, April 1989.

[38] D. Bestion, M. Boucker, P. Boudier, Ph. Fillion, M. Grandotto, A. Guelfi, J.M. Hérard, E. Hervieu, and P. Péturaud. A new multi-scale platform for advanced nuclear Thermal-hydraulics. Proc. in the 11th International Topical Meeting on Nuclear Thermal-Hydraulics (NURETH-11), Avignon (France) 2-6 October, Paper 31, 2005.

[39] P.G. Ciarlet. The Finite Element Method for elliptic problems. Studies in Mathematics and its Applications. North-Holland, first edition, 1978.

[40] M. R. Hestenes and E. L. Stiefel. Methods of conjugate gradients for solving linear systems. J. Res. Nat. Bur. Standards Sect. 5, vol. 49, pp. 409-436, 1952.

[41] P. Sonneveld. CGS: A fast lanczos-type solver for nonsymmetric linear system. SIAM J.Sci. Statist.Comput.10, 36-52, 1989. 